\title{
Implementation of a coupled treatment of the one-turn mapping in the ray-tracing code Zgoubi
}

\author{
F. Desforges
}

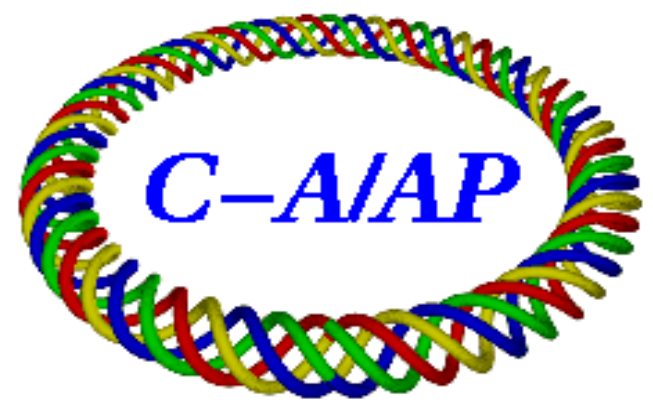

\section{Collider-Accelerator Department Brookhaven National Laboratory Upton, NY 11973}

Notice: This document has been authorized by employees of Brookhaven Science Associates, LLC under Contract No. DE-AC02-98CH10886 with the U.S. Department of Energy. The United States Government retains a nonexclusive, paid-up, irrevocable, world-wide license to publish or reproduce the published form of this document, or allow others to do so, for United States Government purposes. 


\title{
IMPLEMENTATION OF A COUPLED TREATMENT OF THE ONE-TURN MAPPING IN THE RAY-TRACING CODE ZGOUBI
}

August 27, 2012

\author{
FRÉDÉRIC DESFORGES \\ frederic.desforges@grenoble-inp.org \\ Grenoble Institute of Technology, School of Physics, Electronics and Materials \\ Nuclear Sciences and Engineering Department, 2011-2012
}

\author{
Internship supervised by Dr. FrançOIS MÉOT \\ Collider Accelerator Department, Spin group \\ fmeot@bnl.gov \\ Brookhaven National Laboratory \\ P.O. Box 5000 \\ Upton, NY 11973-5000, United States of America
}

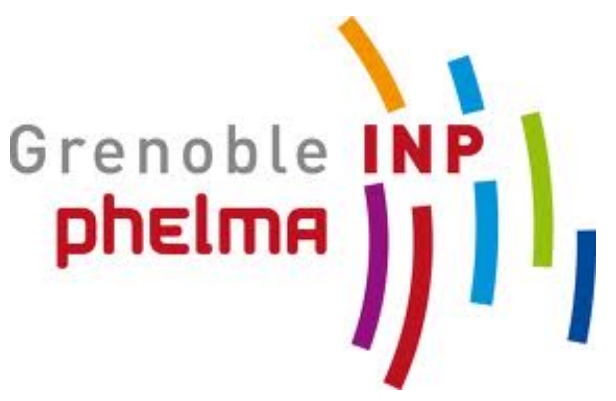




\section{ACKNOWLEDGEMENTS}

I sincerely thank the Brookhaven National Laboratory chair Mr Thomas ROSER, the division head Wolfram FISCHER and my supervisor FrANÇOIS MÉOT for accepting me at BNL and for this opportunity to work in a stimulating framework. I thank FRANÇOIS MÉOT for his scientific explanations and for his patience.

Many thanks to Yann who helped me to install computer tools on my laptop and frequently provided me helpful explanations about accelerator physics as well as computing science. I thank Nicholaos and Leif for their attention and advices during the weekly meetings.

I also want to thank Guillaume, Niccolò, Vincent, Charles, Karim and Zhe for their advices and for our many professional and non-professional talks. It was rewarding and truly made my everyday life more interesting.

Finally, I thank the whole staff of BNL for their cordial welcome. 


\section{Contents}

1 LINEAR TRANSVERSE BEAM DYNAMICS IN CIRCULAR ACCELERATORS 8

1.1 Transverse motions in accelerators and HiLL's equations . . . . . . . . . . . . 8

1.2 General solution of HiLL's equations and TwIss parameters . . . . . . . . . . . . . . 9

1.3 Matrix formalism, Twiss parameters and betatron tune . . . . . . . . . . . . . 10

2 PRELIMINARY STUDY OF THE WEAK LINEAR COUPLING WITH ZGOUBI 11

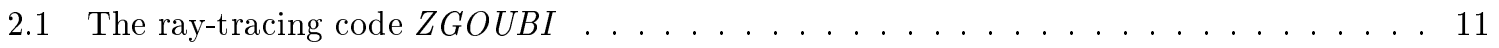

2.2 Benchmark systems $\ldots \ldots \ldots \ldots \ldots \ldots \ldots \ldots \ldots \ldots \ldots$

2.3 Simulations with $Z G O U B D \ldots \ldots \ldots \ldots \ldots \ldots \ldots \ldots$

3 FORMALISM OF THE WEAK LINEAR COUPLING: EDWARDS AND TENG'S

PARAMETRIZATION

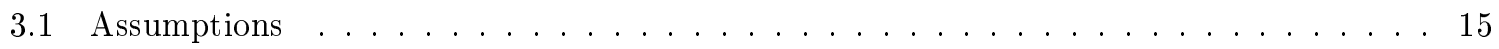

3.2 One-turn mapping and Twiss' matrix in the uncoupled frame. . . . . . . . . . . . . 15

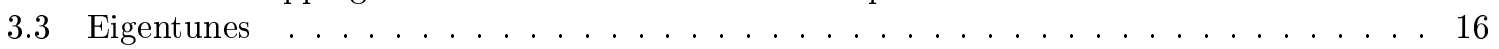

3.4 EDWARDS and TENG's parametrization: generalized TWISS' parameters and coupling

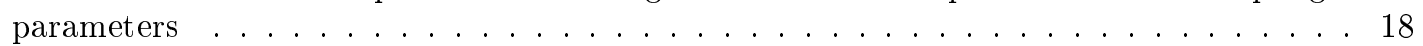

4 EDWARDS AND TENG'S PARAMETRIZATION AND HAMILTONIAN PERTURBATION THEORY FOR THE WEAK LINEAR DIFFERENCE RESONANCES 23

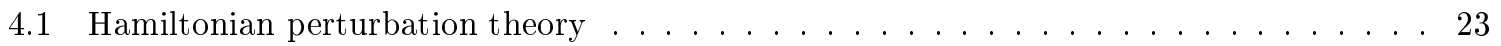

$4.2 \quad$ Hamiltonian perturbation theory parameters, unperturbed tunes and eigentunes . . . . 24

4.3 Hamiltonian perturbation theory parameters as functions of the coupling parameters . . 26

5 EDWARDS AND TENG'S PARAMETRIZATION IMPLEMENTED INTO ZGOUBI 29

$5.1 \quad$ EDWARDS and TENG's parametrization in ForTRAN $77 \ldots \ldots \ldots$. . . . . . . . 29

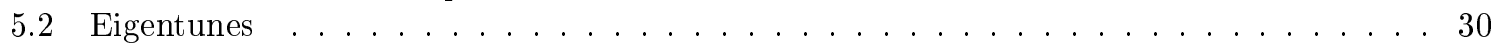

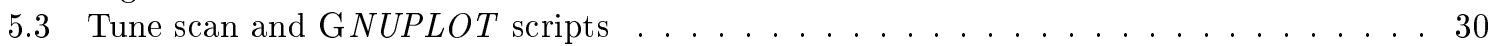

5.4 Tune scan: eigentunes and unperturbed tunes $\ldots \ldots \ldots \ldots \ldots \ldots$

5.5 Hamiltonian perturbation theory parameters $\ldots \ldots \ldots \ldots$. . . . . . . . 33

$5.6 \quad$ Propagation of the generalized TwISs' parameters and the coupling parameter $r$ along the ALTERNATING GRADIENT SYNCHROTRON $\ldots \ldots \ldots \ldots \ldots$ 


\section{INTRODUCTION \& CONTEXT}

The spin is an intrinsic property of elementary particles and is considered as a quantum angular momentum. It is defined by a direction which is either up or down and an absolute value which is proportional to the elementary quantum of action. It has no analogue in classical mechanics but, as the angular momentum in classic mechanics, the spin of composite systems such as the proton is a combination of its spin components. Although knowing the proton components and their spin, their contribution to the proton spin is unknown and the physicists are still trying to unravel the mystery. A recent theoretical work suggests that the contribution carried by the gluons may be large but it has to be confirmed experimentally.

The main accelerator facility which is looking for answers about the origin of the proton spin is located at the Brookhaven National Laboratory (Upton, New York, United States of America). This laboratory has the biggest accelerator complex dedicated to the colliding of polarized proton beam. The protons are first supplied by the Optically Pumped Polarized Ion Source (OPPIS), followed by a 200-MeV LinAC and are then transferred to the Booster SYNCHROTRON. The protons go into the Alternating Gradient Synchrotron (AGS) which accelerate and prepair them to be injected into the Relativistic Heavy Ion Collider (RHIC). Finally the proton beam is stored into the RHIC ring for several hours and supplies collisions in two detectors of particle experiments placed. The data collected are used for studying the decay products of polarized proton collisions.

Although the current RHIC spin experiments are already providing the first information about the proton's spin origin, the complex RHIC is still under improvement. Indeed it is working at minimizing undesirable effects which damage the beam polarization along the acceleration. The polarized proton beam is prone to depolarization due to spin resonances ${ }^{2}$. They could be partially avoided by improving the control of some beam parameters. For instance coupling between the two transverse motions occurs in AGS and modifies the beam parameters predicted by the simulations. The locations of all the coupling sources in the AGS are not really known which makes their removal or compensation difficult to elaborate.

This requires improving the simulations of beam dynamics made in the AGS. They need to be performed with, on one hand, a very accurate model and, on the other hand, a code capable of analyzing a coupled motion. Two codes are mainly used: $M A D X{ }^{3}$ and a ray-tracing code called $Z G O U B I^{4}$ which is developing by Dr. FrançOIs MÉOT. It is scheduled to install ZGOUBI onto the computers of the RHIC complex main control room in order to facilitate and extend its use. Nevertheless $Z G O U B I$ is not completely able to deal with coupled one-turn mapping produced by the ray-tracing so it is necessary to find a way of treating the coupled systems and to implement it within the existing code, that will allow producing coupled optical functions and transporting them using the ray-tracing method.

The purpose of this report is to present the results of my research work performed during this 5month internship. It mainly consisted in searching and synthesizing the appropriate formalism in order to fully treat the coupled which outcomes from $Z G O U B I$. It required to find a way of implementing it into the existing $Z G O U B I$ code then to test it. Finally after validation of these add-ons some simulations have been handled by $Z G O U B I$ for the AGS.

First of all some theoritical elements of linear transverse beam dynamics in circular accelerators will be briefly exposed. Then some simulations handled by ZGOUBI using this data treatment formalism will be performed for uncoupled and coupled benchmark systems. It will allow to simply study how the formalism used by $Z G O U B I$ behaves in presence of a weak and linear coupling. A formalism which

\footnotetext{
${ }^{1}$ For additional information about the Brookhaven National Laboratory and its accelerators, see appendix A.1 and A.2.

${ }^{2}$ Spin tune: number of spin precessions per revolution. More details about the spin tune resonances are given in the appendix B.1.

${ }^{3}$ The MAD-X (Methodical Accelerator Design) program is a general purpose accelerator and lattice design program. It is based on the matrix representation. Official website of MAD-X: http://madx.web.cern.ch/madx/

${ }^{4} Z G O U B I$ : The computer code $Z G O U B I$ calculates trajectories of charged particles in magnetic and electric fields by using a numerical method for integrating the LorENTz equation based on TAYLOR series.
} 
is able to deal with coupled motions will be presented: the EDWARDS and TENG's parametrization. A section of this report is dedicated to a physical interpretation of the EDWARDS and TENG's formalism in the frame of the weak linear difference coupling resonances described by the Hamiltonian perturbation theory. The last section will exhibit the results of simulations testing the validity of the previously proposed formalism. It will end with simulations performed on the AGS and some interesting features which come out the EDWARDS and TENG's formalism. Finally a conclusion and some prospects of my work will be outlined. 


\section{LINEAR TRANSVERSE BEAM DYNAMICS IN CIRCU- LAR ACCELERATORS}

\subsection{Transverse motions in accelerators and HILL's equations}

The transverse motion of particules in circular accelerators will be described in the laboratory referential by small perturbations from the central orbit. This orbit is considered as a equilibrium orbit and can be called the reference orbit. The transverse motion will be derived in a local curvilinear coordinate system $(\mathrm{x}, \mathrm{y}, \mathrm{s})$ that follows the central orbit as shown in the following scheme:

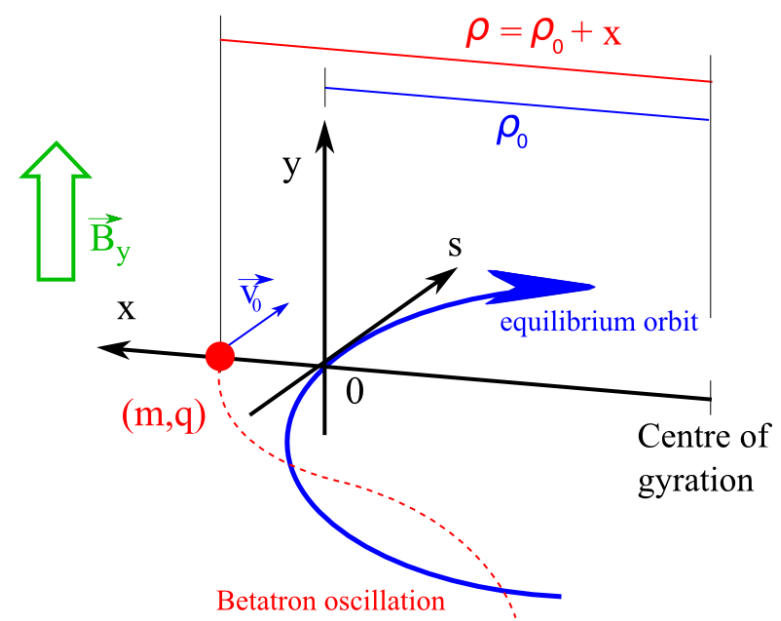

Figure 1.1: Motion of a particule within a circular accelerator described in the laboratory referential with a local curvilinear coordinate system $(x, y, s)$ that follows the equilibrium orbit.

The radii of curvature of the central orbit and the particle trajectory are labeled $\rho_{0}$ and $\rho$, respectively. $q$ is the charge of the particule, $m$ its mass, $v$ its speed and $v_{0}$ its longitudinal component. Only the transverse motion in the plane of bending is going to be fully derived because the calculi are similar for the transverse motion in the plane perpendicular to bending. To this purpose it is requires to assume the paraxial approximation as valid. It means that the deviation from the circular orbit is always small such as the angular velocity can be approximated by $\omega=\frac{v}{\rho} \approx \frac{v_{0}}{\rho}$. Then bending and focusing magnetic elements are assumed as the only components of an accelerator at this stage, so the momentum remains constant along the trajectory. This assumption combined with the paraxial approximation allows to assume $\frac{d}{d t} \approx v_{0} \frac{d}{d s}$. The second NEWTON's law for the LORENTZ force is:

$$
q v_{0} B_{y}=m \frac{d^{2} \rho}{d t^{2}}-m \frac{v_{0}^{2}}{\rho}=m v_{0}^{2} \frac{d^{2} \rho}{d s^{2}}-m \frac{v_{0}^{2}}{\rho}
$$

Making two more approximations will lead to the transverse motion equation. The first approximation consists in expanding the radius of curvature of the particle trajectory. $\rho$ is expandable because it was assumed the perturbations from the central orbit as small compared to the radius of curvature of the central orbit i.e. $x \ll \rho_{0}$. Keeping the two first orders is accurate enough. Moreover it is a linear approximation which will be convenient for future derivations:

$$
\frac{1}{\rho}=\frac{1}{x+\rho_{0}} \approx \frac{1}{\rho_{0}}\left(1-\frac{x}{\rho_{0}}\right)
$$

Then ones expands the vertical magnetic field in a TAYLOR series of $x$ up to the quadrupole component and defines the normalized quadrupole gradient $k$ : 


$$
B_{y}(x) \approx-B_{y}^{0}+\left(\frac{\partial B_{y}}{\partial x}\right)_{x=0} x=-B_{y}^{0}+B_{y}^{0} \rho_{0} k x
$$

where $k$ is the normalized gradient:

$$
k=\frac{1}{B_{y}^{0} \rho_{0}}\left(\frac{\partial B_{y}}{\partial x}\right)_{x=0}
$$

Using that the radius of curvature for the central orbit is $\rho_{0}=\frac{m v_{0}}{q B_{y}^{0}}$ and inserting the equations 1.2 and 1.3 into the equation 1.1 yields the transverse motion equation to be established:

$$
\frac{d^{2} x}{d s^{2}}+K(s) x=0
$$

with $K(s)$ the focusing constant which is equal to $\frac{1}{\rho_{0}^{2}}-k(s)$ in the horizontal plane. The focusing constant is arclength dependent because the magnetic gradient varies along the ring. This equation is also know as the HiLL's equation.

As the curvature of the central orbit is zero in the vertical plane the focusing constant is simply equal to $-k$ for the vertical transverse motion.

\subsection{General solution of HiLL's equations and TwISs parameters}

The Hill equation is a differential equation which describes the transverse motion in circular accelerators. So the lattice and its properties are periodic. Consequently the HiLL's equation has periodic coefficients and thus it exists a pseudo-periodic solution called the betatron oscillation:

$$
\begin{gathered}
u(s)=\sqrt{\varepsilon \beta(s)} \cos \left(\varphi(s)+\varphi_{0}\right) \\
\frac{d u}{d s}(s)=-\sqrt{\frac{\varepsilon}{\beta(s)}}\left[\alpha(s) \cos \left(\varphi(s)+\varphi_{0}\right)+\sin \left(\varphi(s)+\varphi_{0}\right)\right]
\end{gathered}
$$

with $u$ a transverse coordinate, either $x$ or $y$, and:

- $\beta(s)$ the betatron function, $\alpha(s)=-\frac{1}{2} \frac{d \beta(s)}{d s}$ the alpha function and $\gamma(s)=\frac{1+\alpha(s)^{2}}{\beta(s)}$ the gamma functions. They are known as the Twiss parameters.

- $\varphi(s)=\int_{0}^{s} \frac{d \tilde{s}}{\beta(\tilde{s})}$ the phase of the particle and $\varphi_{0}$ a constant phase determined by the initial conditions. The number of transverse oscillations around the equilibrium orbit per turn, i.e. betatron oscillations, is usually called the betatron tune and is determined by $Q=\frac{1}{2 \pi} \oint \frac{d \tilde{s}}{\beta(\tilde{s})}$.

- $\varepsilon$ the emittance which is also known as the Courant \& SNyder invariant. This motion invariant times $\pi$ equals the area of the ellipse described by the betatron motion in phase space. All ions in the beam will have a value for this invariant and follow similar ellipses. One notices that $\sqrt{\varepsilon \beta(s)}$ is the amplitude of the motion equation 1.6 so one can interpret the square-root of the betatron function as the transverse envelope of the beam.

The trajectory of a particle in a transverse phase space has been plotted: 


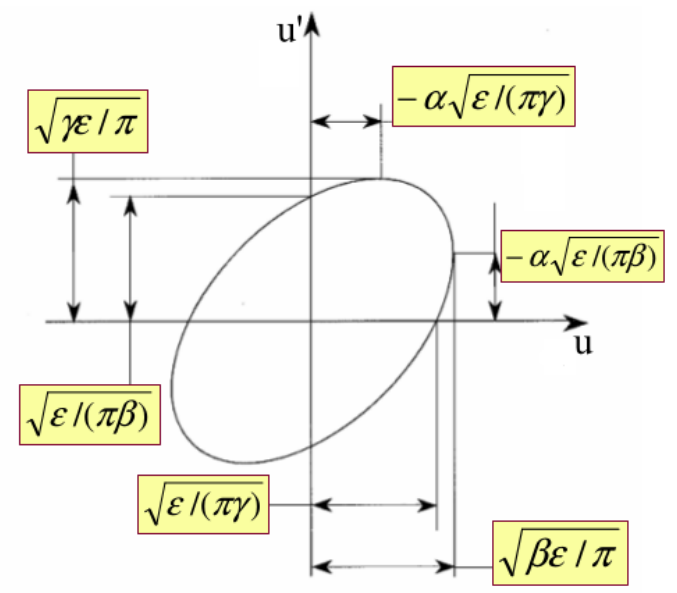

Area $=\varepsilon=\pi\left(\gamma \mathrm{u}^{2}+2 \alpha \mathrm{u}^{\prime} \mathrm{u}+\beta \mathrm{u}^{\prime^{2}}\right)$

Figure 1.2: Trajectory of a particle in a transverse phase space

\subsection{Matrix formalism, Twiss parameters and betatron tune}

Another approach is to use matrices for transporting the phase space coordinates $\vec{X}=\left[\begin{array}{c}x \\ x^{\prime} \\ y \\ y^{\prime}\end{array}\right]$ along the ring. If the initial coordinates are known as well as the transfer matrix $\mathcal{T}_{0 \rightarrow 1}$ from the initial location at $s_{0}$ to a further location at $s_{1}$, it possible to obtain the coordinates at $s_{1}$ :

$$
\vec{X}_{1}=\mathcal{T}_{0 \rightarrow 1} \vec{X}_{0}
$$

The one-turn mapping is defined as the $4 \times 4$ transfer matrix, at a point in the laboratory frame, for one turn around the system:

$$
\mathcal{T}=\left[\begin{array}{cccc}
t_{11} & t_{12} & t_{13} & t_{14} \\
t_{21} & t_{22} & t_{23} & t_{24} \\
t_{31} & t_{32} & t_{33} & t_{34} \\
t_{41} & t_{42} & t_{43} & t_{44}
\end{array}\right]
$$

In absence of coupling between the horizontal and the vertical betatron oscillations the coefficients of the anti-diagonal $2 \times 2$ matrices are equal to zero. The one-turn matrix of a periodic lattice without coupling in the laboratory frame could be identified as follows [1]:

$$
\mathcal{M}_{\text {uncoupled }}=\left[\begin{array}{cc}
\mathcal{A}_{x} & 0 \\
0 & \mathcal{A}_{y}
\end{array}\right]
$$

where

$$
\mathcal{A}_{i \in\{x, y\}}=\left[\begin{array}{cc}
\cos 2 \pi Q_{i}+\alpha_{i} \sin 2 \pi Q_{i} & \beta_{i} \sin 2 \pi Q_{i} \\
-\gamma_{i} \sin 2 \pi Q_{i} & \cos 2 \pi Q_{i}-\alpha_{i} \sin 2 \pi Q_{i}
\end{array}\right]
$$

with $\beta_{i}, \alpha_{i}, \gamma_{i}$ the Twiss parameters and $Q_{i}$ the betatron tune presented in the previous subsection. 


\section{PRELIMINARY STUDY OF THE WEAK LINEAR COU- PLING WITH ZGOUBI}

\subsection{The ray-tracing code $Z G O U B I$}

$Z G O U B I$ is a FORTRAN77 code which calculates trajectories of charged particles in magnetic and electric fields by using a numerical method for integrating the LORENTZ equation based on TAYLOR series [14. In addition to the ray-tracing particle, $Z G O U B I$ is also able to perform a lot of various analyzes: betatron tunes, Twiss' parameters, synchrotron radiation... As these features are too numerous to be described one is going to briefly explain the numerical process used for ray-tracing particles in accelerators.

The particle ray-tracing process of $Z G O U B I$ is based on the taylor expansion of the position $\vec{R}\left(M_{1}\right)$ and the velocity $\vec{u}\left(M_{1}\right)$ at point $M_{1}$ from initial position $\vec{R}\left(M_{0}\right)$ and unit velocity $\vec{u}\left(M_{0}\right)=\frac{\vec{v}\left(M_{0}\right)}{v\left(M_{0}\right)}$ following a displacement $\Delta s$ :

$$
\begin{gathered}
\vec{R}\left(M_{1}\right)=\vec{R}\left(M_{0}\right)+\sum_{n=1}^{\infty} \frac{\Delta s^{n}}{n !} \vec{R}^{(n)}\left(M_{0}\right) \approx \vec{R}\left(M_{0}\right)+\sum_{n=1}^{6} \frac{\Delta s^{n}}{n !} \vec{u}^{(n-1)}\left(M_{0}\right) \\
\vec{u}\left(M_{1}\right)=\vec{u}\left(M_{0}\right)+\sum_{n=1}^{\infty} \frac{\Delta s^{n}}{n !} \vec{u}^{(n)}\left(M_{0}\right) \approx \vec{u}\left(M_{0}\right)+\sum_{n=1}^{5} \frac{\Delta s^{n}}{n !} \vec{u}^{(n)}\left(M_{0}\right)
\end{gathered}
$$

where $\vec{u}^{(n)}\left(M_{0}\right)=\frac{d^{n} \vec{u}\left(M_{0}\right)}{d s^{n}}$ is obtained from the LoRENTz $\left(\vec{u}^{\prime}\left(M_{0}\right) \propto \vec{u}\left(M_{0}\right) \times \vec{B}\left(M_{0}\right)\right)$ equation and the magnetic fields, possibly using field maps. Moreover the truncation of TAYLOR expansions are obtained considering the compromise between accuracy and speed of computations. The numerical principle is graphically resumed by the following scheme:

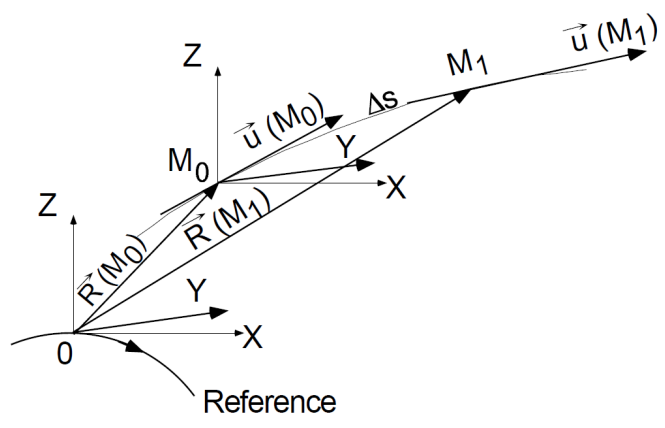

Figure 2.1: Position and motion of a particle modeled by Zgoubi code

\subsection{Benchmark systems}

Benchmark systems need to be developed in order to study the weak linear coupling.

First it requires to develop them by pair such that each pair contains one system without weak linear coupling and one with. Consequently it will allow to compare a coupled system with its uncoupled counterpart. Then the benchmarking of the implementation of the Edwards and Teng's formalism into $Z G O U B I$ will require systems with fractional tunes inferior and superior to 0.5 . The way of computing the tunes will depend on the value of their fractional part.

For fulfilling all of these requirements two pairs of systems have been developed. In this study the benchmark systems are circular systems without defects and so the dispersion as well as the 
chromaticity effect: 5 will not be considered.

First a FODO cell (system 1) and a FODO cell with a skew quadrupole (system 2) have been designed for describing the case when the fractional part of the tune is inferior to 0.5 :
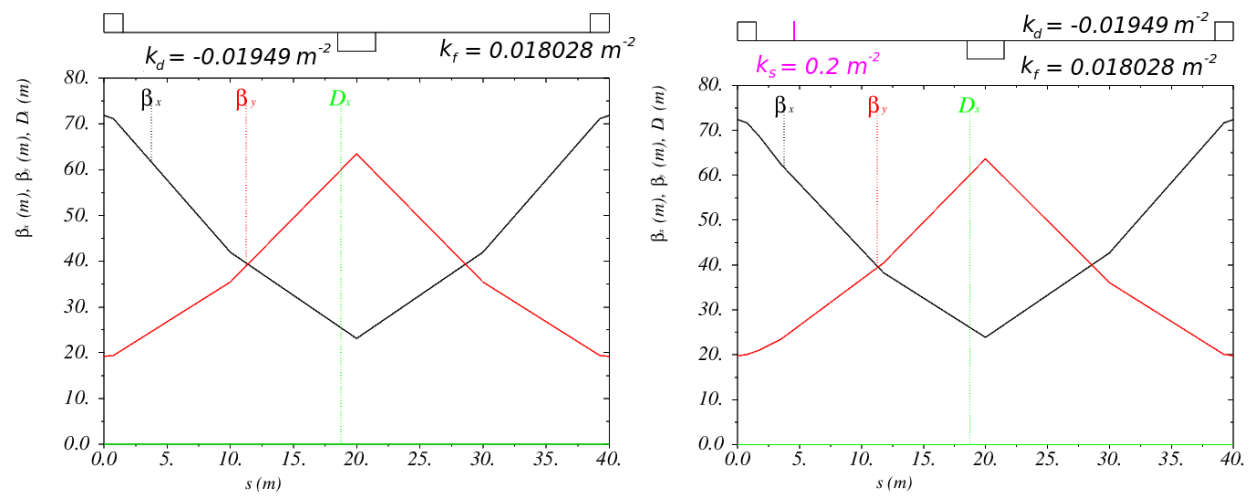

Figure 2.2: The system 1 (left scheme) is a FODO cell. The strength of the defocusing quadrupole is $k_{d}=-0.01949 \mathrm{~m}^{-2}$ and the strength of the focusing quadrupole is $k_{f}=0.018028 \mathrm{~m}^{-2}$. Their length is $l_{\text {quad }}=3 \mathrm{~m}$. The system 2 (right scheme) represents the same FODO cell plus a skew quadrupole which has been added at $4.97 \mathrm{~m}$. The strength of the skew quadrupole is $k_{s}=0.2 \mathrm{~m}^{-2}$ and its length is $l_{\text {skew }}=0.03 \mathrm{~m}$.

Then 5 FODO cells similar to the system 1 have been designed both without (system 3 ) and with (system 4) one skew quadrupole for describing the case when the fractional part of the tune is superior to 0.5 :
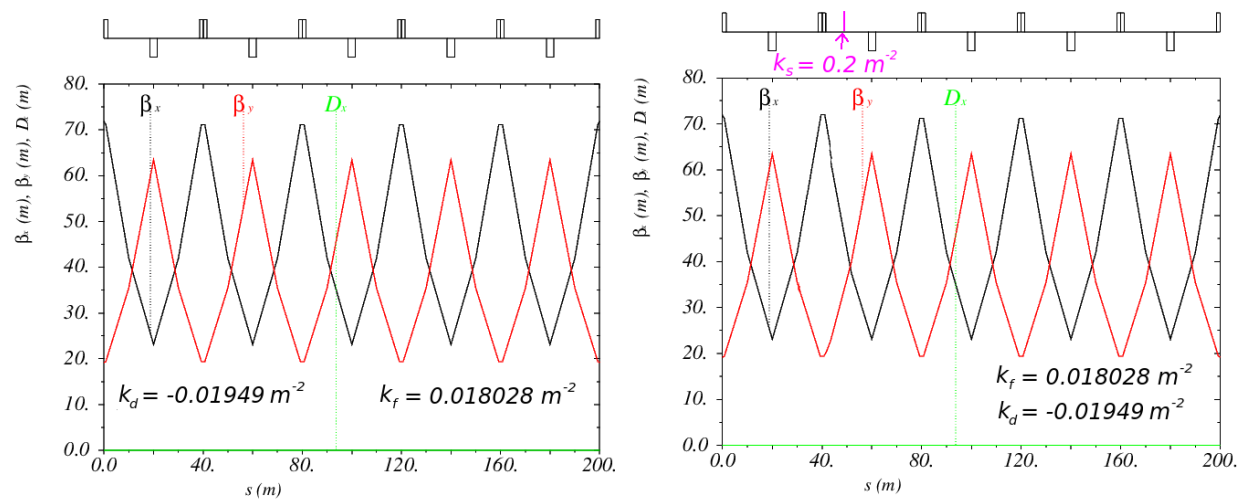

Figure 2.3: The system 3 (left scheme) consists of 5 FODO cells. The strength of the defocusing quadrupole is $k_{d}=-0.01949 \mathrm{~m}^{-2}$ and the strength of the focusing quadrupole is $k_{f}=0.018028 \mathrm{~m}^{-2}$. Their length is $l_{\text {quad }}=3 \mathrm{~m}$. The system 4 represents four regular FODO cells similar to the system 1 plus one system 2 . The strength of the skew quadrupole is $k_{s}=0.2 \mathrm{~m}^{-2}$ and its length is $l_{\text {skew }}=0.03 \mathrm{~m}$.

\subsection{Simulations with $Z G O U B I$}

Simulations of these systems have been handled with $Z G O U B I$ for a $450 \mathrm{GeV}$ proton beam. Two different ways of computing the betatron tunes have been used. Actually the tunes cannot be fully calculated. Indeed the numerical process which consists in considering the infinite set of solutions of

\footnotetext{
${ }^{5}$ Dispersion and chromaticity: They are both chromatic effects. A distribution in momentum will result in, on one hand, a central orbit distribution i.e. dispersion and in, on another hand, a tune distribution i.e. chromaticity.
} 
$\cos \left(2 \pi Q_{i}\right)=a_{i}$ yields only the fractional part of the tune to be determined. The terms betatron tunes, eigentunes or tunes will refer to their fractional part in future considerations.

1. ZGOUBI is able to establish the $6 \times 6$ coupled one-turn mapping. In the uncoupled hypothesis. $Q_{y}$ and $Q_{x}$ can be calculated from this output matrix by considering it equal to a two-dimensional TwISS matrix . $Q_{y}^{Z G O U B I}$ and $Q_{x}^{Z G O U B I}$ will refer to the values computed by this analytical identification.

2. $Z G O U B I$ is also able to handle the ray-tracing of particles. An off-centered particle has performed 500 turns into each system and $Z G O U B I$ has recorded its transverse position every turn at the entry of the systems. $Q_{y}^{D F T}$ and $Q_{x}^{D F T}$ will refer to the values computed owing to a discrete FOURIER transform (DFT) of this discrete set of values. Indeed a particle behaving under the betatron phenomenon is oscillating around the closed orbit at the betatron frequency $f_{i}=2 \pi Q_{i \in\{x, y\}}$. Consequently a DFT yields the betatron frequencies and so the tunes to be determined from the transverse positions recorded during the ray-tracing. An example of analysis for the systems 1 and 3 of have been plotted:

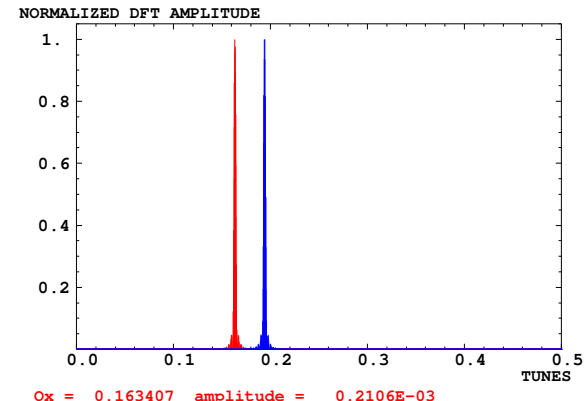

8x $\equiv 8: 163907$ amplitude $\equiv 8: 2105 \mathrm{E}-03$

Figure 2.4: Tunes calculated by a discrete FOURIER transform of the 500-turns ray-tracing of an off-centered particle. It has been plotted for the uncoupled system 1. The different colors refer to different set of values. The red and blue graphs are the discrete Fourier transform in the horizontal and vertical planes, respectively.
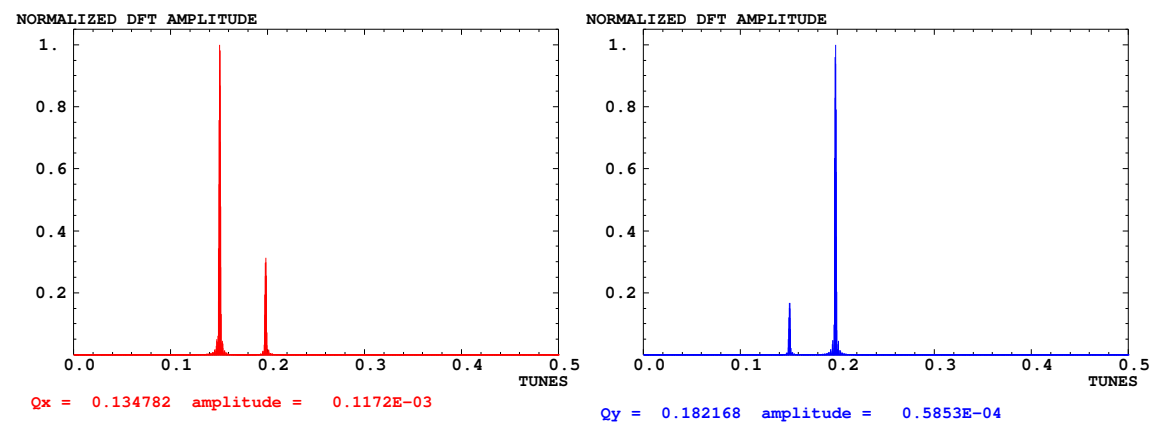

Figure 2.5: Vertical tunes calculated by a discrete FoURIER transform of the 500-turns ray-tracing of an off-centered particle. It has been plotted for the coupled system 3 . The different colors refer to different set of values. The red and blue graphs are the discrete FouRIER transform in the horizontal and vertical planes, respectively.

The figure 2.5 shows a consequence of the coupling. It makes the two betatron oscillations correlated. The betatron oscillation which only requires to be described by one betatron frequency in the 
uncoupled case is now a motion composed of two modes. The mode with the highest normalized DFT amplitude is called the main mode. Its contribution in amplitude of oscillation is more important than the other mode. $Q_{y}^{D F T}$ and $Q_{x}^{D F T}$ will refer to the main mode in the vertical and horizontal direction, respectively.

The values obtained by the two methods for the four systems are summarized in the following table:

\begin{tabular}{|c|c|c|c|c|c|}
\hline & & $Q_{y}^{D F T}$ & $Q_{y}^{Z G O U B I}$ & $Q_{x}^{D F T}$ & $Q_{x}^{Z G O U B I}$ \\
\hline \hline \multirow{2}{*}{ No Coupling } & system 1 & 0.193996 & 0.194001 & 0.163407 & 0.163407 \\
\cline { 2 - 6 } & system 3 & 0.970017 & 0.970006 & 0.817038 & 0.817035 \\
\hline \multirow{2}{*}{ Coupling } & system 2 & 0.182168 & 0.173906 & 0.134782 & 0.144466 \\
\cline { 2 - 6 } & system 4 & 0.960226 & 0.956709 & 0.800959 & 0.801919 \\
\hline
\end{tabular}

Table 2.1: Comparison of the non-integer part of the horizontal betatron tune $Q_{x}$ and the vertical one $Q_{y} . Q_{y}^{Z G O U B I}$ and $Q_{x}^{Z G O U B I}$ refer to the tunes calculated from the elements of the diagonal $2 \times 2$ transfer matrix, at a point in the laboratory frame, for one turn around the system. $Q_{y}^{D F T}$ and $Q_{x}^{D F T}$ refer to the tunes calculated by a discrete FouRIER transform of the 500-turns ray-tracing of a non-centered particle. It appears discrepancies when the coupling is introduced into the FODO cells.

In absence of coupling (system $1 \& 3$ ) the two processes are equivalent and give the same results. However in presence of coupling (system $2 \& 4$ ) it is no longer true. Considering what is done experimentally gives a hint to foresee which method is correct.

The experimental method for measuring the tunes is based on a DFT: the center of mass of the bunch which is initially on the central orbit is excited by an electromagnetic kick, the transverse position at a certain point is recorded turn-by-turn by a Beam Position Monitor (BPM) then this set of values is analyzed by a DFT. The outputs of this method per planes are two modes of which the main one is called the betatron tune. This process is prefered by the operators of the RHIC complex for measuring the tunes along the acceleration process because it is intrinsically not affected by the coupling.

The discrepancies shown in the table 2.1 lead to reconsider the mathematical process used for computing the tunes from the output matrix of $Z G O U B I$ when the system is coupled. Some codes developed for the accelerator physics such as $M A D X$ fully analyzes the coupling by using a formalism which is not used in the matrix derivations from ray-tracing by $Z G O U B I$ : the EDWARDS and TENG's parametrization. This formalism will be presented in the following section. It will be explained how the motion can be parametrized from the output of $Z G O U B I$ : the one-turn mapping. The formalism proposed has been applied into a program written in ForTRAN77. The results of the simulations will be described in the last section. The aim of this program is to give to ZGOUBI a way of analyzing the coupled motion without using a DFT. It has to be enough flexible in order to be integrated into the existing $Z G O U B I$ code. 


\section{FORMALISM OF THE WEAK LINEAR COUPLING: ED- WARDS AND TENG'S PARAMETRIZATION}

\subsection{Assumptions}

The following assumptions are necessary for developing the formalism proposed by EDWARDS and TEng:

- Linear beam dynamics. The systems are conservative (time independent) so all the transformations along the ring will be symplectic $[8]$.

- Weak and linear coupling.

- Floquet resonances ${ }^{6}$ excluded.

All of these assumptions are realistic for the ray-tracing of particles in the benchmark systems. Indeed the skew quadrupole is a linear optical element and its strength used is one tenth of the regular quadrupole strength. Integer and half-integer betatron tunes provoke FLOQUET's resonances so this case does not need to be considered. This fact will be taken into account to determine the tunes of coupled systems.

It is interesting to notice that the linear coupling introduced by the snakes into the AGS is weak so the formalism developed for the preliminary study on the FODO cells could be applied to the AGS.

\subsection{One-turn mapping and TwISs' matrix in the uncoupled frame}

In presence of coupling one can no longer make the identification between the two matrices $\mathcal{M}_{\text {uncoupled }}$ and $\mathcal{T}$ made in the uncoupled case presented in the subsection 1.3. Indeed the coefficients of the antidiagonal $2 \times 2$ matrices can be different from zero so the regular formalism is no longer appropriate for describing the motion of particle. Nevertheless one would like to keep the form of the transfer matrix in order to describe the two decoupled motions by making an analogy with the uncoupled case. The uncoupled formalism can be extended to a decoupled one by changing the referential in which the transfer matrix is written [2, 3, 4, 5]. It yields the tunes and the TwIss' parameters along the new axes to be defined such that the one-turn matrix is written :

$$
\mathcal{M}_{\text {decoupled }}=\left[\begin{array}{cc}
\mathcal{A}_{1} & 0 \\
0 & \mathcal{A}_{2}
\end{array}\right]=\mathcal{V}^{-1} \mathcal{T} \mathcal{V}
$$

where the index 1 and 2 refer to the two eigenmodes, $\mathcal{V}$ is the transformation matrix $\left(\vec{X}_{\text {uncoupled }}=\mathcal{V} \vec{X}_{\text {coupled }}\right)$ and $V^{-1}$ its inverse matrix. The energy of the system, i.e. its volume in the phase space, has to be the same in both frames so:

$$
\operatorname{det}(\mathcal{V})=1
$$

The $2 \times 2$ matrices are defined by:

$$
\mathcal{A}_{i \in\{1,2\}}=\left[\begin{array}{cc}
\cos 2 \pi Q_{i}+\alpha_{i} \sin 2 \pi Q_{i} & \beta_{i} \sin 2 \pi Q_{i} \\
-\gamma_{i} \sin 2 \pi Q_{i} & \cos 2 \pi Q_{i}-\alpha_{i} \sin 2 \pi Q_{i}
\end{array}\right]
$$

In this case the $Q_{i}$ are named the eigentunes and $\alpha_{i}, \beta_{i}$ and $\gamma_{i}$ the generalized Twiss parameters. The physical meaning of the eigentunes and the generalized Twiss parameters are quite different from the uncoupled ones. For instance the phase advance is now fed with two modes and there is no longer a single oscillation frequency corresponding to the betatron frequency of an off-centered particle. They only have a similar meaning in a transformed referential which is physically meaningless.

\footnotetext{
${ }^{6}$ FlOQUET resonances: Resonances due to dipole and quadrupole imperfections. They correspond to integer and half-integer betatron tunes.
} 
The analytical process for determining the eigentunes, the generalized Twiss' parameters and the transformation matrix will be describe in the subsection 3.4 .

\subsection{Eigentunes}

Assuming $\mathcal{T}$ symplectic yields the one-turn mapping to have four eigenvalues which are complex conjugate two by two [2]:

$$
\mathcal{T}=\mathcal{E} D \mathcal{E}^{-1}
$$

with $\mathcal{D}$ the diagonal matrix of the eigenvalues:

$$
\mathcal{D}=\left[\begin{array}{cccc}
\lambda_{1} & 0 & 0 & 0 \\
0 & \lambda_{1}^{*} & 0 & 0 \\
0 & 0 & \lambda_{2} & 0 \\
0 & 0 & 0 & \lambda_{2}^{*}
\end{array}\right]
$$

and $\mathcal{E}=\left[\overrightarrow{e_{1}}, \overrightarrow{e_{1}^{*}}, \overrightarrow{e_{2}}, \overrightarrow{e_{2}^{*}}\right]$ the matrix of the normalized eigenvectors.

The symplectic condition imposes $\operatorname{det}(\mathcal{T})=1$ so there are four different theoritical cases:

1. All four eigenvalues lie on the unit circle, forming two complex conjugate and reciprocal pairs.

2. One reciprocal pair is real, the others are complex and on the unit circle.

3. Two real reciprocal pairs.

4. One eigenvalue complex $\lambda_{1}=\lambda$ and not on the unit circle; the other eigenvalues must then be $\lambda_{1}^{*}=\lambda^{*}, \lambda_{2}=\frac{1}{\lambda}$ and $\lambda_{2}^{*}=\frac{1}{\lambda^{*}}$.

Having a stable system requires the trace of the matrix to be strictly inferior to 2 in absolute value. In addition the motion is unstable if any eigenvalue is greater than 1 in absolute value. These last conditions and the symplectic condition are summarized by the two following equations:

$$
\left\{\begin{array}{l}
|\operatorname{Tr}(\mathcal{T})|=\left|\lambda_{1}+\lambda_{1}^{*}+\lambda_{2}+\lambda_{2}^{*}\right|=\left|\mathscr{R} e\left(\lambda_{1}\right)+\mathscr{R} e\left(\lambda_{2}\right)\right|<2 \\
\operatorname{det}(\mathcal{T})=\lambda_{1} \lambda_{1}^{*} \lambda_{2} \lambda_{2}^{*}=\left|\lambda_{1}\right|^{2}\left|\lambda_{2}\right|^{2}=1
\end{array}\right.
$$

One deduces from a disjunction elimination that these conditions of stability and the exclusion of FLOQUET's resonances (subsection 3.1) yields only the situation (1) to be stable. Cases (1), (2), and (3) correspond to the uncoupled case with both modes stable, one mode stable and one unstable, and two unstable modes, respectively. Case (4) represents a case generated by the coupling when the eigenvalues for the unperturbed modes are such as $\cos \left(2 \pi Q_{x}^{0}\right) \approx \cos \left(2 \pi Q_{y}^{0}\right)$. As $\lambda$ is not on the unit circle at least one eigenvalue is greater than 1 in absolute value so one mode is unstable. All the cases $\cos \left(2 \pi Q_{x}^{0}\right) \approx \cos \left(2 \pi Q_{y}^{0}\right)$ can be equally described by $Q_{x}^{0} \pm Q_{y}^{0}=p \in \mathbb{N}$ and are called linear coupling resonances. $Q_{x}^{0}+Q_{y}^{0}=p$ corresponds to the linear sum coupling resonances and $Q_{x}^{0}-Q_{y}^{0}=p$ the linear difference coupling resonances. Additional explanations will be given by the subsection 4.1 .

As only the situation (1) is stable the absolute value of the eigenvalues is equal to 1 and so they can be written by using the exponential form: $\lambda_{i \in\{1,2\}}=\exp \left(i 2 \pi Q_{i}\right)$. So 3.5 becomes:

$$
\mathcal{D}=\left[\begin{array}{cccc}
\exp \left(i 2 \pi Q_{1}\right) & 0 & 0 & 0 \\
0 & \exp \left(-i 2 \pi Q_{1}\right) & 0 & 0 \\
0 & 0 & \exp \left(i 2 \pi Q_{2}\right) & 0 \\
0 & 0 & 0 & \exp \left(-i 2 \pi Q_{2}\right)
\end{array}\right]
$$

The diagonalization of the matrix $\mathcal{T}$ does not directly lead the eigentunes to be determined. It is impossible to compute the eigentunes from the complex eigenvalues because one does not know 
if $\lambda_{1}=\exp \left(i 2 \pi Q_{1}\right)$ or $\exp \left(-i 2 \pi Q_{1}\right)$ i.e. $Q_{1}=\frac{1}{2 \pi} \arg \left(\lambda_{1}\right)$ or $Q_{1}=1-\frac{1}{2 \pi} \arg \left(\lambda_{1}\right)$. Despite this indetermination, a process can be used for determining the eigentunes. Some considerations taken into account by this method need to be detailed:

- The linear coupling resonances $Q_{x}^{0} \pm Q_{y}^{0}=p \in \mathbb{N}$ will not be considered because it leads to a case which cannot be solved by that manner. It is suitable at the vicinity of the resonance and not on the resonance.

- One has assumed that the coupling is weak then the coupled transfer matrix and its eigenvalues differ only slightly from the unperturbed transfer matrix and the unperturbed eigenvalues, respectively.

- One has already excluded the case where the uncoupled system is near a FLOQUET resonance.

The following graph summarizes these three considerations required for understanding the process:

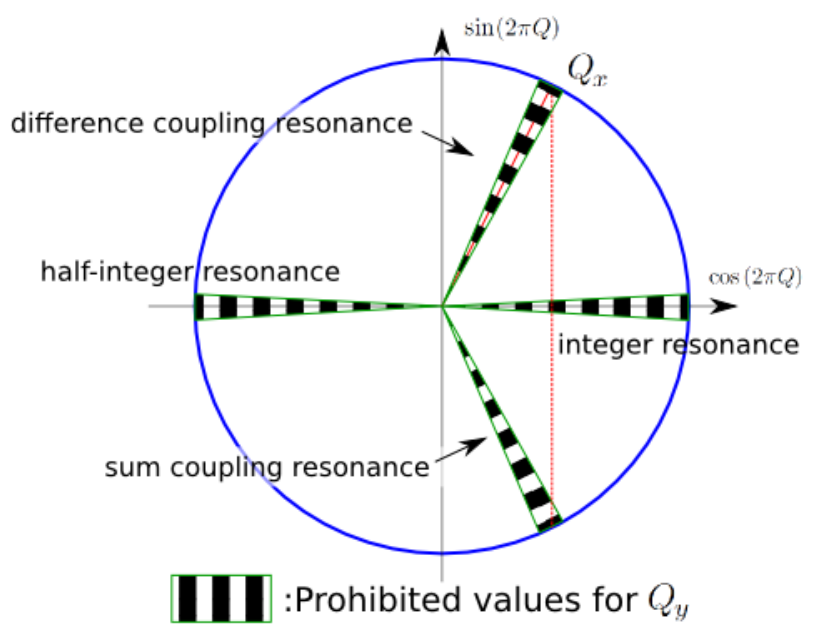

Figure 3.1: Prohibited values of the tunes reported on the trigonometric circle. The variation of the tunes are treated following the perturbation theory under the assumptions of a weak and linear coupling. $Q_{x}$ and $Q_{y}$ are the horizontal betatron tune and the vertical one, respectively.

The prohibited area for $Q_{y}^{0}$ means the set of values for which $Q_{x}^{0}$ and $Q_{y}^{0}$ are not computable with the process that will be presented. The one located in the upper right quarter of the circle represents the linear difference coupling resonances. The area located in the lower right quarter of the circle represents the sum difference coupling resonances.

Considering these requirements yields the indetermination of the eigentunes to be computed as follows:

1. Computation of the unperturbed tunes $Q_{x}^{0}$ and $Q_{y}^{0}$ by identifying $\mathcal{T}$ to $\mathcal{M}_{\text {uncoupled }}$ even if $t_{13}$, $t_{14}, t_{31} \ldots \neq 0$.

2. Computation of $\cos \left(2 \pi Q_{1}\right)$ and $\cos \left(2 \pi Q_{2}\right)$ from the complex eigenvalues of $\mathcal{T}$.

3. Comparison of the two possible values of $Q_{1}$ to both $Q_{x}^{0}$ and $Q_{y}^{0}$ in order to know which value of $Q_{1}$ is the perturbed value of $Q_{x}^{0}$ or $Q_{y}^{0}$. Idem for $Q_{2}$.

The process works because the particular case of the coupling resonances is prohibited as it has been simply exhibited by the previous scheme. $Q_{1}$ and $Q_{2}$ no longer refer to the perturbed value of $Q_{x}^{0}$ or $Q_{y}^{0}$ when the linear coupling resonance is reached: $Q_{x}^{0} \pm Q_{y}^{0}=p \in \mathbb{N}$. In this particular case both of the eigentunes refer to both $Q_{x}^{0}$ and $Q_{y}^{0}$ because the proportions of the two modes in both planes are equal. 


\subsection{EDWARDS and TENG's parametrization: generalized TwISs' parameters and coupling parameters}

EDWARds and TEng proposed to parametrize the transformation matrix as follows [2] :

$$
\mathcal{V}=\left[\begin{array}{cc}
r \mathcal{I} & \mathcal{C} \\
-\mathcal{C}^{+} & r \mathcal{I}
\end{array}\right]
$$

where $I$ is the identity matrix, $r$ the coupling parameter, $\mathcal{C}=\left[\begin{array}{ll}c_{11} & c_{12} \\ c_{21} & c_{22}\end{array}\right]$ the coupling matrix and its sympletic conjugate $\mathcal{C}^{+}=\left[\begin{array}{cc}c_{22} & -c_{12} \\ -c_{21} & c_{11}\end{array}\right]$. It is imposed that $\operatorname{det}(\mathcal{V})=r^{2}+\operatorname{det}(\mathcal{C})=1$ because it is required by the equation $3.2, r$ is taken positive in [3, 4, 5, 6, so one will keep this convention for the next analytical developments. In the following parts of this report the EDWARDS and TENG's parameters will refer to both the generalized Twiss' parameters and the coupling parameters $r$ and $\mathcal{C}$.

All of these parameters as well as the generalized Twiss' parameters can be derived from the eigenvectors of the transfer matrix. However the analytical process establishing a relation between both the coupling/generalized Twiss' parameters and the eigenvectors is not direct. It requires first to consider the action-angle frame then to define a transformation matrix from this frame to the laboratory one (subsubsection 3.4.1). It will be a powerful intermediate tool for determining the generalized Twiss' parameters and the coupling parameters (subsubsection 3.4.2 and 3.4.3). The physical meaning of these parameters will be detailed in the section 4 .

\subsubsection{Action-angle frame}

For general two-dimensional linearly coupled motion, single-particle motion can be written through the action-angle frame as [3]:

$$
\left(\begin{array}{c}
x \\
x^{\prime} \\
y \\
y^{\prime}
\end{array}\right)=P\left(\begin{array}{c}
\sqrt{2 J_{1}} \cos \phi_{1} \\
-\sqrt{2 J_{1}} \sin \phi_{1} \\
\sqrt{2 J_{2}} \cos \phi_{2} \\
-\sqrt{2 J_{2}} \sin \phi_{2}
\end{array}\right)
$$

where $\phi_{i}$ and $J_{i}$ are the one-turn phase advance and the constant action of the $i$-eigenmode, respectively. $\mathcal{P}$ is the transformation matrix from the action-angle frame to the laboratory one. The action-angle frame can be seen as a normalized frame in which the motion over one turn is describe as a combination of two rotations: first a $\phi_{1}$-angle rotation in the eigen-phase space of the mode 1 then a $\phi_{2}$-angle rotation in the eigen-phase space of the mode 2 (see figure 3.2 ).

The $4 \times 4$ transfer matrix, at a certain point in the action-angle frame, for one turn around the system is:

$$
\mathcal{R}\left(\triangle \phi_{1}, \triangle \phi_{2}\right)=\left[\begin{array}{cccc}
\cos \triangle \phi_{1} & \sin \triangle \phi_{1} & 0 & 0 \\
-\sin \triangle \phi_{1} & \cos \triangle \phi_{1} & 0 & 0 \\
0 & 0 & \cos \triangle \phi_{2} & \sin \triangle \phi_{2} \\
0 & 0 & -\sin \triangle \phi_{2} & \cos \triangle \phi_{2}
\end{array}\right]=\mathcal{P}^{-1} \mathcal{T} \mathcal{P}
$$

where $\triangle \phi_{i}$ is the eigen phase advance of the mode $i$ after one turn around the system and $\mathcal{P}^{-1}$ the inverse matrix of $\mathcal{P}$. Considering the definition of the eigentunes $Q_{i}=\frac{\Delta \phi_{i}}{2 \pi}$ yields the equation 3.10 to become:

$$
\mathcal{R}\left(2 \pi Q_{1}, 2 \pi Q_{2}\right)=\left[\begin{array}{cccc}
\cos 2 \pi Q_{1} & \sin 2 \pi Q_{1} & 0 & 0 \\
-\sin 2 \pi Q_{1} & \cos 2 \pi Q_{1} & 0 & 0 \\
0 & 0 & \cos 2 \pi Q_{2} & \sin 2 \pi Q_{2} \\
0 & 0 & -\sin 2 \pi Q_{2} & \cos 2 \pi Q_{2}
\end{array}\right]
$$




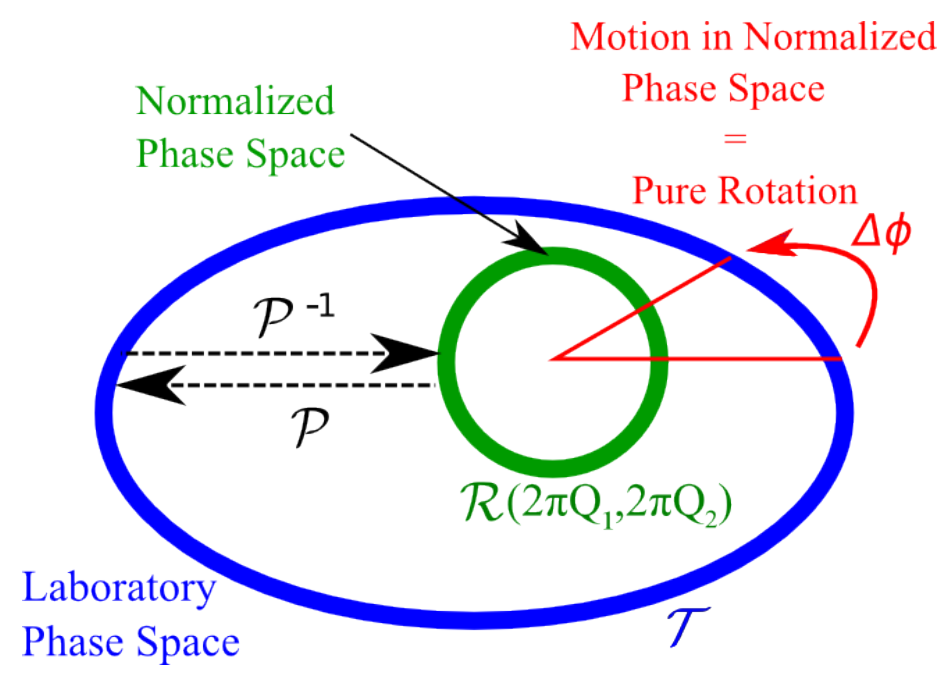

Figure 3.2: Particle trajectory in phase space of the action-angle frame (green curve) and the laboratory frame (blue curve). The $4 \times 4$ transfer matrix, at a certain point in the action-angle frame, for one turn around the system is $\mathcal{R}\left(2 \pi Q_{1}, 2 \pi Q_{2}\right)$. The one-turn mapping is $\mathcal{T}$. $\mathcal{P}$ is the transformation matrix from the action-angle frame to the laboratory frame and $\mathcal{P}^{-1}$ its inverse matrix.

Using this normalized referential helps to notice how close is the previous matrix to the diagonal matrix $\mathcal{D}$ (equation 3.7) and to the generalized Twiss' formalism matrix $\mathcal{M}_{\text {decoupled }}$ (equation 3.1).

If these two frames are slightly changed one should be able to find a relation :

- between the action-angle frame and the eigenmode frame.

- between the action-angle frame and the EDWARDS and TENG's parametrization frame.

Consequently a relation between the eigenmode frame and the EDWARDS and TENG's parametrization frame could be established. A term-by-term identification resulting from this relation could yield the generalized Twiss' parameters and the coupling parameters to be fully determined from the eigenvalues and eigenvectors of the transfer matrix in the laboratory frame. In other words the generalized TwIss' parameters and the coupling parameters could be directly computed from the one-turn mapping. That is convenient because it is what $Z G O U B I$ computes.

\subsubsection{Matrix $\mathcal{P}$, generalized TwISs' parameters and coupling parameters}

$\mathcal{A}_{1}$ and $\mathcal{A}_{2}$ presented in the subsection 3.2 are parametrized using the CouRANT-SNYDER parametrization. It is possible to write them differently in such a way that reveals the formalism used in the action-angle frame:

$$
\mathcal{A}_{i \in\{1,2\}}=\mathcal{U}_{i} \mathcal{R}_{i}\left(2 \pi Q_{i}\right) \mathcal{U}_{i}^{-1}
$$

That is:

$\left[\begin{array}{cc}\cos 2 \pi Q_{i}+\alpha_{i} \sin 2 \pi Q_{i} & \beta_{i} \sin 2 \pi Q_{i} \\ -\gamma_{i} \sin 2 \pi Q_{i} & \cos 2 \pi Q_{i}-\alpha_{i} \sin 2 \pi Q_{i}\end{array}\right]=\left[\begin{array}{cc}\sqrt{\beta_{i}} & 0 \\ \frac{-\alpha_{i}}{\sqrt{\beta_{i}}} & \frac{1}{\sqrt{\beta_{i}}}\end{array}\right]\left[\begin{array}{cc}\cos 2 \pi Q_{i} & \sin 2 \pi Q_{i} \\ -\sin 2 \pi Q_{i} & \cos 2 \pi Q_{i}\end{array}\right]\left[\begin{array}{cc}\frac{1}{\sqrt{\beta_{i}}} & 0 \\ \frac{\alpha_{i}}{\sqrt{\beta_{i}}} & \sqrt{\beta_{i}}\end{array}\right]$

Writing $\mathcal{U}=\left[\begin{array}{cc}\mathcal{U}_{1} & 0 \\ 0 & \mathcal{U}_{2}\end{array}\right]$ as the transformation matrix from the eigenmode frame to the actionangle frame yields a relation between $\mathcal{T}$ and $\mathcal{R}$ : 


$$
\mathcal{T}=\mathcal{V U \mathcal { R }}\left(2 \pi Q_{1}, 2 \pi Q_{2}\right) \mathcal{U}^{-1} \mathcal{V}^{-1}
$$

Comparing the equations 3.10 and 3.13 gives:

$$
\mathcal{P}=\mathcal{V U}
$$

On one hand the determinant of $\mathcal{P}$ is derived from the determinants of $\mathcal{V}$ and $\mathcal{U}$. $\operatorname{det}(\mathcal{U})=1$ by definition of $\mathcal{U}$ and $\operatorname{det}(\mathcal{V})=1$ according to the EDWARDS and TENG's parametrization, so:

$$
\operatorname{det}(P)=\operatorname{det}(U) \times \operatorname{det}(V)=1
$$

On another hand $V U$ is expanded into generalized Twiss' parameters and coupling parameters:

$$
\mathcal{P}=\left(\begin{array}{cccc}
r \sqrt{\beta_{1}} & 0 & c_{11} \sqrt{\beta_{2}}-\frac{c_{12} \alpha_{2}}{\sqrt{\beta_{2}}} & \frac{c_{12}}{\sqrt{\beta_{2}}} \\
-\frac{\alpha_{1} r}{\sqrt{\beta_{1}}} & \frac{r}{\sqrt{\beta_{1}}} & c_{21} \sqrt{\beta_{2}}-\frac{c_{22} \alpha_{2}}{\sqrt{\beta_{2}}} & \frac{c_{22}}{\sqrt{\beta_{2}}} \\
-\frac{c_{12} \alpha_{1}}{\sqrt{\beta_{1}}}-c_{22} \sqrt{\beta_{1}} & \frac{c_{12}}{\sqrt{\beta_{1}}} & r \sqrt{\beta_{2}} & 0 \\
\frac{c_{11} \alpha_{1}}{\sqrt{\beta_{1}}}+c_{21} \sqrt{\beta_{1}} & -\frac{c_{11}}{\sqrt{\beta_{1}}} & -\frac{\alpha_{2} r}{\sqrt{\beta_{2}}} & \frac{r}{\sqrt{\beta_{2}}}
\end{array}\right)
$$

Consequently the generalized Twiss' parameters and coupling parameters can be obtained from the matrix $P$ coefficients:

$$
\begin{array}{r}
\left\{\begin{array}{c}
\alpha_{1}=-\frac{p_{21}}{p_{22}} \\
\beta_{1}=\frac{p_{11}}{p_{22}} \\
\gamma_{1}=\frac{p_{21}^{2}+p_{22}^{2}}{p_{11} p_{22}}
\end{array}\right. \\
\left\{\begin{array}{c}
\alpha_{2}=-\frac{p_{43}}{p_{44}} \\
\beta_{2}=\frac{p_{33}}{p_{44}} \\
\gamma_{2}=\frac{p_{43}^{2}+p_{44}^{2}}{p_{33} p_{44}}
\end{array}\right. \\
r=\sqrt{p_{11} p_{22}}=\sqrt{p_{33} p_{44}} \\
\mathcal{C}=r\left[\begin{array}{ll}
p_{11} & p_{12} \\
p_{21} & p_{22}
\end{array}\right]\left[\begin{array}{cc}
p_{44} & -p_{34} \\
-p_{43} & p_{33}
\end{array}\right]
\end{array}
$$

A relation between the EDWARDS and TENG's parameters and the matrix $\mathcal{P}$ is now well established. The next step consists in constructing the matrix $\mathcal{P}$ from the eigenvectors of the one-turn mapping $\mathcal{T}$. Consequently passing by this matrix $\mathcal{P}$ will make possible the analytical determination of the generalized TwISS' parameters and coupling parameters from the output matrix of ZGOUBI: the one-turn mapping.

\subsubsection{Matrix $\mathcal{P}$ and eigenvectors}

The four normalized eigenvectors of the one-turn mapping $\mathcal{T}$ at one point are $\overrightarrow{e_{1}}, \overrightarrow{e_{1}^{*}}, \overrightarrow{e_{2}}, \overrightarrow{e_{2}^{*}}$ such that:

$$
\left\{\begin{array}{c}
\mathcal{T} \overrightarrow{e_{1}}=\exp \left(i 2 \pi Q_{1}\right) \overrightarrow{e_{1}} \\
\mathcal{T} \overrightarrow{e_{1}^{*}}=\exp \left(-i 2 \pi Q_{1}\right) \overrightarrow{e_{1}^{*}} \\
\mathcal{T} \overrightarrow{e_{2}}=\exp \left(i 2 \pi Q_{2}\right) \overrightarrow{e_{2}} \\
\mathcal{T} \overrightarrow{e_{2}^{*}}=\exp \left(-i 2 \pi Q_{2}\right) \overrightarrow{e_{2}^{*}}
\end{array}\right.
$$

Substituting the equation 3.11 into 3.21 gives:

$$
\left\{\begin{array}{c}
\mathcal{R}\left(2 \pi Q_{1}, 2 \pi Q_{2}\right) \mathcal{P}^{-1} \overrightarrow{e_{1}}=\exp \left(i 2 \pi Q_{1}\right) \mathcal{P}^{-1} \overrightarrow{e_{1}} \\
\mathcal{R}\left(2 \pi Q_{1}, 2 \pi Q_{2}\right) \mathcal{P}^{-1} \overrightarrow{e_{1}^{*}}=\exp \left(-i 2 \pi Q_{1}\right) \mathcal{P}^{-1} \overrightarrow{e_{1}^{*}} \\
\mathcal{R}\left(2 \pi Q_{1}, 2 \pi Q_{2}\right) \mathcal{P}^{-1} \overrightarrow{e_{2}}=\exp \left(i 2 \pi Q_{2}\right) \mathcal{P}^{-1} \overrightarrow{e_{2}} \\
\mathcal{R}\left(2 \pi Q_{1}, 2 \pi Q_{2}\right) \mathcal{P}^{-1} \overrightarrow{e_{2}^{*}}=\exp \left(-i 2 \pi Q_{2}\right) \mathcal{P}^{-1} \overrightarrow{e_{2}^{*}}
\end{array}\right.
$$


Consequently $\mathcal{P}^{-1} \overrightarrow{e_{i}}$ are eigenvectors of $\mathcal{R}\left(2 \pi Q_{1}, 2 \pi Q_{2}\right)$. Besides $\mathcal{R}\left(2 \pi Q_{1}, 2 \pi Q_{2}\right)$ has four normalized and two by two orthogonal eigenvectors, one decides to impose the $\overrightarrow{e_{i}}$ such as:

$$
\left\{\begin{array}{l}
\mathcal{P}^{-1} \overrightarrow{e_{1}}=\frac{1}{\sqrt{2}}\left[\begin{array}{cccc}
-i & 1 & 0 & 0
\end{array}\right]^{T} \\
\mathcal{P}^{-1} \overrightarrow{e_{1}^{*}}=\frac{1}{\sqrt{2}}\left[\begin{array}{llll}
i & 1 & 0 & 0
\end{array}\right]^{T} \\
\mathcal{P}^{-1} \overrightarrow{e_{2}}=\frac{1}{\sqrt{2}}\left[\begin{array}{llll}
0 & 0 & -i & 1
\end{array}\right]^{T} \\
\mathcal{P}^{-1} \overrightarrow{e_{2}^{*}}=\frac{1}{\sqrt{2}}\left[\begin{array}{llll}
0 & 0 & i & 1
\end{array}\right]^{T}
\end{array}\right.
$$

So:

which is equivalent to:

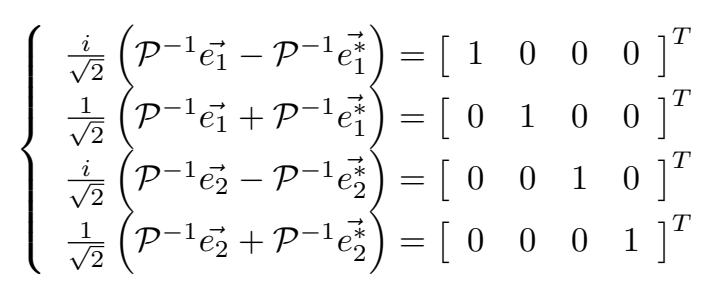

$$
\mathcal{P}=\mathcal{E} \mathcal{G}
$$

with:

$$
\mathcal{G}=\frac{\sqrt{2}}{2}\left[\begin{array}{cccc}
i & 1 & 0 & 0 \\
-i & 1 & 0 & 0 \\
0 & 0 & i & 1 \\
0 & 0 & -i & 1
\end{array}\right]
$$

This choice is arbitrary, it will not influence the final result. Although $\mathcal{P}$ seems to be determined by the previous equations two more modifications have to be implemented in order to make the termby-term identification possible.

First the requirement of a determinant equal to one (equation 3.15 imposes to divide the eigenvectors by their determinant.

Then the original matrix eigenvectors $\mathcal{E}$ have to be modified in order to assure $p_{12}=p_{34}=0[3$ :

$$
\mathcal{H}=\left[\begin{array}{cccc}
\exp \left(-i \frac{\pi}{2}-i \theta_{1}\right) & 0 & 0 & 0 \\
0 & \exp \left(-i \frac{\pi}{2}+i \theta_{1}\right) & 0 & 0 \\
0 & 0 & \exp \left(-i \frac{\pi}{2}-i \theta_{2}\right) & 0 \\
0 & 0 & 0 & \exp \left(-i \frac{\pi}{2}+i \theta_{2}\right)
\end{array}\right]
$$

with $\theta_{1}=\arg \left(\overrightarrow{e_{1}}\right)$ and $\theta_{2}=\arg \left(\overrightarrow{e_{2}}\right)$.

So after these two modifications the eigenvectors matrix becomes:

$$
\mathcal{F}=\left[\overrightarrow{f_{1}}, \overrightarrow{f_{1}^{*}}, \overrightarrow{f_{2}}, \overrightarrow{f_{2}^{*}}\right]=\operatorname{det}(\mathcal{E})^{-\frac{1}{4}} \mathcal{H E}
$$

Finally the relation between the matrix $\mathcal{P}$ and the normalized eigenvectors of the one-turn mapping $\mathcal{T}$ is:

$$
\mathcal{P}=\operatorname{det}(\mathcal{E})^{-\frac{1}{4}} \mathcal{H E} \mathcal{G}=\mathcal{F} \mathcal{G}
$$

which is equivalent to:

$$
\mathcal{P}=\left[\frac{i}{\sqrt{2}}\left(\overrightarrow{f_{1}}-\overrightarrow{f_{1}^{*}}\right), \frac{1}{\sqrt{2}}\left(\overrightarrow{f_{1}}+\overrightarrow{f_{1}^{*}}\right), \frac{i}{\sqrt{2}}\left(\overrightarrow{f_{2}}-\overrightarrow{f_{2}^{*}}\right), \frac{1}{\sqrt{2}}\left(\overrightarrow{f_{2}}+\overrightarrow{f_{2}^{*}}\right)\right]
$$


This relation shows that the real and imaginary parts of the eigenvectors are proportional to reference vectors of the angle-action frame. Moreover it yields the coupling parameters and the generalized TwIss' matrix to be fully determined from the diagonalization of the one-turn mapping i.e. the $Z G O U B I$ output matrix. Their physical meaning will be studied in the section 4.

\subsubsection{Propagation of the matrix $\mathcal{P}$, the coupling parameters and the generalized TwISs' parameters}

The $4 \times 4$ transfer matrix $\mathcal{T}_{1}$, at a point labeled 1 in the laboratory frame, for one turn around the system can be transported to another point labeled 2. It requires the transfert matrix from the starting point to the ending point $\mathcal{T}_{1 \rightarrow 2}$ to be known beforehand. Indeed the next formula gives the relation between the two transfer matrices:

$$
\mathcal{T}_{2}=\mathcal{T}_{2 \rightarrow 1} \mathcal{T}_{1} \mathcal{T}_{1 \rightarrow 2}=\mathcal{T}_{1 \rightarrow 2}^{-1} \mathcal{T}_{1} \mathcal{T}_{1 \rightarrow 2}
$$

In addition it has been explained in the subsection 3.3 that the transfer matrices can be diagonalized:

$$
\mathcal{E}_{2}^{-1} \mathcal{D}_{2} \mathcal{E}_{2}=\left(\mathcal{E}_{1} \mathcal{T}_{1 \rightarrow 2}\right)^{-1} \mathcal{D}_{1}\left(\mathcal{E}_{1} \mathcal{T}_{1 \rightarrow 2}\right)
$$

As the matrix diagonalization leads to a single characterisation, i.e. $\mathcal{D}_{2}=\mathcal{D}_{1}$, and the matrix product is not commutative it is possible to make an identification on each side of the equality. It

finally yields the transported eigenvectors to be determined from the starting point eigenvectors and the transfert matrix from 1 to 2 :

$$
\mathcal{E}_{2}=\mathcal{E}_{1} \mathcal{T}_{1 \rightarrow 2}
$$

Consequently the computation of the transported coupling and generalized Twiss' parameters can be easily done by applying the procedures of the previous subsubsection to these transported eigenvectors. 


\section{EDWARDS AND TENG'S PARAMETRIZATION AND HAMIL- TONIAN PERTURBATION THEORY FOR THE WEAK LINEAR DIFFERENCE RESONANCES}

\subsection{Hamiltonian perturbation theory}

The Hamiltonian perturbation theory of the weak and linear coupling is developed in [7] and gives the Hamiltonian of the perturbation induced by a weak linear coupling :

$\mathscr{H}_{1}(s)=\mathscr{H}_{1}^{\text {sum }}(s)+\mathscr{H}_{1}^{\text {dif }}(s)=\frac{L}{2 \pi} \sum_{l=-1,+1} \sum_{k=-\infty}^{+\infty} C_{k, l}(s) \sqrt{a_{x}(s) a_{y}(s)} \cos \left(\psi_{x}(s)+l \psi_{y}(s)+\Delta_{k, l} \frac{2 \pi}{L} s\right)$

where $L$ is the length of the lattice, $l$ is a parameter for differenciating two types of resonances: $l=+1$ for the sum resonances and $l=-1$ for the difference resonances, the $a_{i}$ are the coupled motion's amplitudes, the $\psi_{i}$ are the coupled motion's phases, $s$ is the distance between the skew quadrupole and the reference point, $\Delta_{k, l}$ is the fractional uncoupled tune split: $\Delta_{k, l}=Q_{x}^{0}+l Q_{y}^{0}-k N$ with $N$ the superperiodicity of the system.

$k$ is the index of the Fourier serie and the $C_{k, l}$ are its coefficients. The use of a Fourier serie imposes the system to have periodic betatron functions so this formalism should be used in periodic systems. These coefficients are also called the coupling coefficients and are defined as:

$$
C_{k, l}(s)=\frac{1}{2 \pi} \int_{0}^{L} \sqrt{\beta_{x}(\widetilde{s}) \beta_{y}(\widetilde{s})} k_{s}(\widetilde{s}) e^{i\left(\Psi_{x}(s, \widetilde{s})+l \Psi_{y}(s, \widetilde{s})-\Delta_{k, l} \frac{2 \pi}{L} \widetilde{s}\right)} d \widetilde{s}
$$

where the $\beta_{i}$ are the unperturbed betatron functions, the $\Psi_{i}$ are the unperturbed betatron phase advances between $s$ and $\widetilde{s}, k_{s}$ is the strength of the skew quadrupole.

According to [7] most of the terms of the FouRIER serie oscillate too fast for jeopardizing the stability of the particle. Only one term is slowly varying, that is when $k=m$ such as $\Delta_{m, l} \approx 0 \Leftrightarrow$ $m \approx \frac{Q_{x}^{0}+l Q_{y}^{0}}{N}$.

The future analytical developments will only deal with weak linear difference resonances because it will be easier for the interpretation of the coupling parameter $r$ and the coupling matrix $\mathcal{C}$. Besides the difference resonances can be tolerated in an accelerator because they do not lead directly to an unstable situation. In the vicinity of a difference resonance the transverse emittances can vary under the effects of coupling but their sum remains constant. Consequently if one is increased the other one has to decrease so their values are physically limited to $\varepsilon_{x}^{0}+\varepsilon_{y}^{0}$. On contrary the linear sum resonances are dangerous for the stability of the beam. Indeed the difference of the transverse emittances has to be conserved so both the vertical and horizontal emittances can diverge [1, 7]. Nevertheless the difference resonances can also be a threat for the beam. The coupling can sometimes shift the tune to the vicinity of a FLOQUET resonance, that is why the coupling effects have to be predicted in order to correct them. A formalism is going to be presented for this purpose.

If only the linear difference resonances are considered the equation 4.1 becomes:

$$
\mathscr{H}_{-}(s)=\frac{L}{2 \pi} C_{-}(s) \sqrt{a_{x}(s) a_{y}(s)} \cos \left(\psi_{x}(s)-\psi_{y}(s)+\Delta_{-} \frac{2 \pi}{L} s\right)
$$

where $\Delta_{-}=Q_{x}^{0}-Q_{y}^{0}-p$ with $p=m N \in \mathbb{N}$. The equation 4.2 becomes:

$$
C_{-}(s)=\left|C_{-}\right| e^{i \chi}=\frac{1}{2 \pi} \int_{0}^{L} \sqrt{\beta_{x}(\widetilde{s}) \beta_{y}(\widetilde{s})} k_{s}(\widetilde{s}) e^{i\left(\Psi_{x}(s, \widetilde{s})-\Psi_{y}(s, \widetilde{s})-\Delta_{-} \frac{2 \pi}{L} \widetilde{s}\right)} d \widetilde{s}
$$


where $\chi$ is the phase of the complex $C_{-}$.

Some linear resonances have been drawn in a tune diagram in order to illustrate the physical meaning of $\Delta_{-}$and $\Delta_{+}=Q_{x}^{0}+Q_{y}^{0}-p$. They represent for the unperturbed system the distance in tune from the nearest linear difference and sum resonance, respectively. Their range is clearly $\left[-\frac{\sqrt{2}}{2} ; \frac{\sqrt{2}}{2}\right]$ and their sum is always inferior to $\sqrt{2}$ in absolute value. The sign of $\Delta_{+/-}$indicates the position of the system relative to the nearest resonance. For example if $\Delta_{+/-}<0$ it means the system is closer to the upper resonance than the lower one. Or, in the context of a tune scan with increase of only $Q_{y}^{0}$, it can also be seen such as the system has not reached the resonance yet.

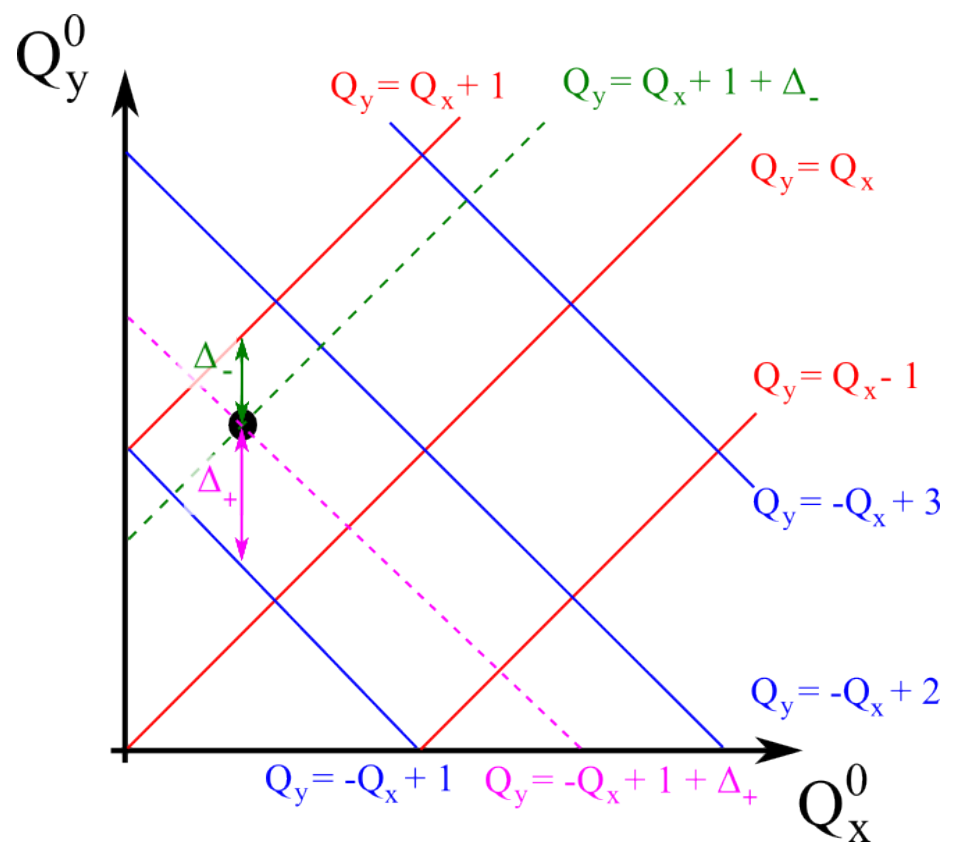

Figure 4.1: Example of tune diagram $\left(Q_{x}^{0}, Q_{y}^{0}\right)$ with $Q_{x}^{0}$ and $Q_{y}^{0}$, respectively . $\Delta_{-}$and $\Delta_{+}$represent the distance in tune from the nearest linear difference and sum resonance, respectively.

\subsection{Hamiltonian perturbation theory parameters, unperturbed tunes and eigentunes}

The Hamiltonian perturbation approach introduces the variables $C_{-}$and $\Delta_{-}$in order to describe the linear weak coupling. This approach will also define the vertical and horizontal unperturbed tunes. One will impose for the following derivations that:

1. $Q_{1}$ is the perturbed value of $Q_{x}^{0}$.

2. $Q_{2}$ is the perturbed value of $Q_{y}^{0}$.

This convention will have consequences on the behaviour of the eigentunes as well as the range of $r$.

The mathematical expressions of $\left|C_{-}\right|$and $\Delta_{-}$+ are not so convenient for getting the physics observed during the tune measurements. It could be easier to interpret them by considering their relation with both the eigentunes and the unperturbed tunes. It has been demonstrated in [7] that: 


$$
\left\{\begin{array}{l}
Q_{1}=Q_{x}^{0}+\frac{\Delta_{-}}{2}-\operatorname{sign}\left(\Delta_{-}\right) \times \frac{1}{2} \sqrt{\Delta_{-}^{2}+\left|C_{-}\right|^{2}} \\
Q_{2}=Q_{y}^{0}-\frac{\Delta_{-}}{2}+\operatorname{sign}\left(\Delta_{-}\right) \times \frac{1}{2} \sqrt{\Delta_{-}^{2}+\left|C_{-}\right|^{2}}
\end{array}\right.
$$

where $\operatorname{sign}\left(\Delta_{-}\right)=\frac{\Delta_{-}}{\left|\Delta_{-}\right|}= \pm 1$.

In absence of coupling $Q_{x}^{0}=Q_{1}$ and $Q_{y}^{0}=Q_{2}$ so the previous formulae yield the coupling strength $\left|C_{-}\right|$to be zero. In addition one notices that $\left|Q_{1}-Q_{2}\right|=\left|C_{-}\right|$when the system is on the resonance i.e. $Q_{x}^{0}=Q_{y}^{0}$.

Plotting the $Q_{1}$ and $Q_{2}$ as a function of $\Delta_{-}$is more convenient for their physical interpretation. Experimentally the following graph could be obtained by making change the strength of a quadrupole which changes the focusing properties of the lattice and so the tunes. All the values of $\Delta_{-}$are thereby scanned:

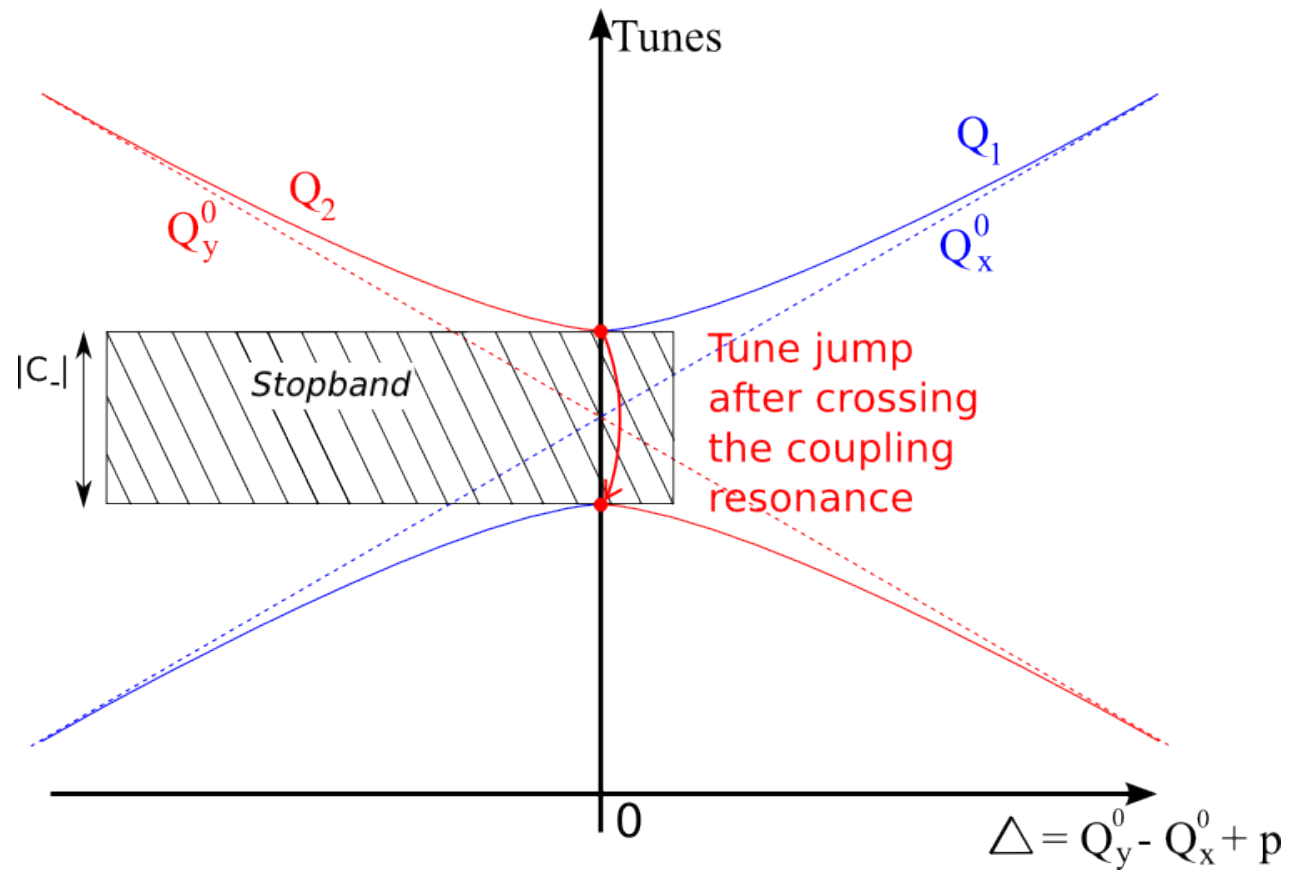

Figure 4.2: Effect of weak linear coupling on tune measurements. The lines are what is really measurable and the dash lines are what should be measured in absence of coupling. $\Delta_{-}$is the tune distance from the resonance which could be scanned by making change the strength of a quadrupole. In the vicinity of the coupling resonance, that is $\Delta_{-} \approx 0$, a stopband with a width of $\left|C_{-}\right|$for the eigentunes appears.

The eigentunes resulting of a weak linear coupling are plotted in lines. The asymptotes of the eigentunes are draw in dash lines and match with the unperturbed tunes. Only the eigenmodes are experimentally observed and it is meaningless to deal with horizontal and vertical tunes when there is coupling.

This graph could help the interpretation of $\Delta_{-}$and $\left|C_{-}\right|$.

On one hand $\Delta_{-}$is simply the tune distance from the resonance where the effects of the coupling are maximum: the frequency split is at its maximum and is entirely due to the coupling coefficient. It means that the further away from the resonance the system is the weaker the coupling effect on the tunes is.

On another hand $\left|C_{-}\right|$could be seen as the width of the eigentune stopband. The stronger the coupling is the higher the difference between the perturbed and unperturbed tunes is thus the larger 
the stopband is.

The "tune jump" displayed on the previous graph must not be interpreted as a physical tune jump resulting from an energy change of the system. This jump is due to a change of the optics and a convention of notations. Indeed one imposed $Q_{1}$ and $Q_{2}$ to correspond to the weakly perturbed $Q_{x}^{0}$ and $Q_{y}^{0}$, respectively. Experimentally two continuous curves are observed without distinction of $Q_{1}$ and $Q_{2}$. The tune jump is only the result of a convention for the notations used. The following schematic DFT illustrates this ambiguity:

$\triangle<0$

\section{Horizontal DFT amplitude}

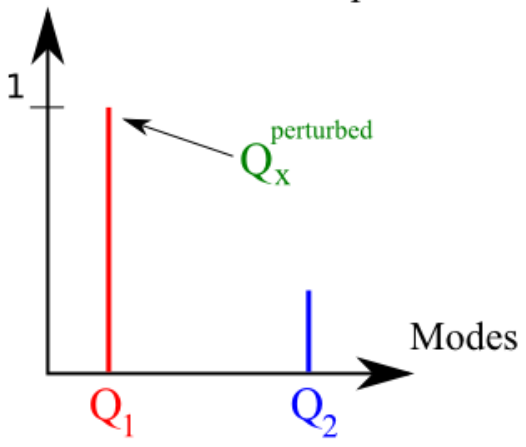

$\triangle=0$<smiles>OC12CC1C2</smiles>
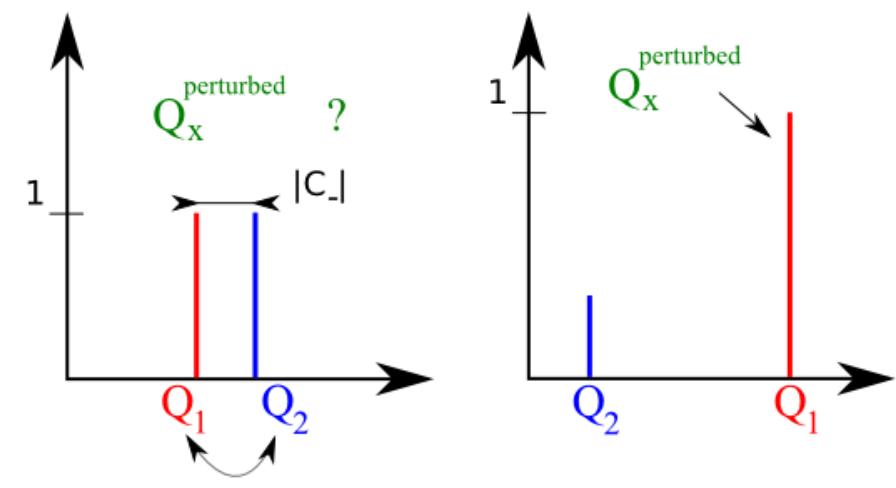

Exchange after crossing the resonance

Figure 4.3: Illustration of the ambiguity of the "tune jump" introduced by the convention of the notations. A DFT is made in the $x$-plane. The perturbed tune $Q_{x}^{\text {perturbed }}$ is the eigentune with the highest DFT amplitude, that is here $Q_{1} . Q_{x}^{\text {perturbed }}$ refers to the main mode in the $x$-plane. $Q_{x}^{\text {perturbed }}$ is undetermined for $\Delta_{-}=0$.

\subsection{Hamiltonian perturbation theory parameters as functions of the cou- pling parameters}

After some algebraic calculations made in [6] and [4 it has been proved that $r>1$ corresponds to a case where the system is closer to a linear sum resonance than a linear difference resonance. The case described by $0<r<1$ is the opposite situation. One can already conclude that $r$ informs us whether the system is working near a linear sum resonance or a linear difference one.

Moreover it is shown in these publications that the parameters developed in the Hamiltonian perturbation approach for the linear difference resonances can be written as a function of the coupling parameters $r$ and $\mathcal{C}$ :

$$
\left\{\begin{aligned}
\left|C_{-}\right| & =2 r \sqrt{1-r^{2}}\left|Q_{1}-Q_{2}-p\right| \\
\Delta_{-} & =\left(2 r^{2}-1\right)\left(Q_{1}-Q_{2}-p\right) \\
\chi & =\arctan \left(\frac{-c_{12}}{\alpha_{1} c_{12}+\beta_{1} c_{22}}\right) \\
\pi-\chi & =\arctan \left(\frac{c_{12}}{-\alpha_{2} c_{12}+\beta_{2} c_{11}}\right)
\end{aligned}\right.
$$

The matrix coeffcients $c_{i j}$ are the normalized real and imaginary part of $\left|C_{-}\right|$(see equation 4.4): 


$$
\left\{\begin{array}{l}
\sin (\chi)=-c_{12} \\
\cos (\chi)=\alpha_{1} c_{12}+\beta_{1} c_{22}
\end{array}\right.
$$

This equation imposes $c_{12}$ to be between -1 and 1 .

Two particular cases come out of these previous equations.

First, according to the equations 4.5 the coupling strength $\left|C_{-}\right|$is zero in absence of coupling so the equations 4.6 gives $r=1$ for an uncoupled system. The equations 4.6 give for the uncoupled systems $\Delta_{-}=Q_{1}-Q_{2}-p=Q_{x}^{0}-Q_{y}^{0}-p$ as expected.

Secondly according to the equations 4.5 the coupling strength and the distance from the linear difference resonance at the resonance are $\left|C_{-}\right|=\left|Q_{1}-Q_{2}-p\right|$ and $\Delta_{-}=0$, respectively. The equations 4.6 indicates that these values are reached for $r=\frac{\sqrt{2}}{2}$.

Knowing $r$ indicates whether the system is coupled and whether it is on the resonance. Neverthless it is interesting to notice that $r$ does not directly inform how far of the resonance a coupled system is. $\Delta_{-}$is the key factor for describing the distance in tune from the resonance. As it has been exhibited in the graph 4.1 the sign of $\Delta_{-}$reveals whether the relative position to the nearest coupling resonance. This feature of the formalism will help to determine the range of $r$. Considering the scheme 4.2 and the convention previously chosen (see subsection 4.2 imposes $\frac{\sqrt{2}}{2} \leq r \leq 1$. Indeed $\Delta_{-}$as well as $Q_{1}-Q_{2}$ is negative in the left part of the graph and positive in its right part. consequently it is required that $2 r^{2}-1 \geq 0$, i.e. $r \geq \frac{\sqrt{2}}{2}$, whatever the optics is.

As a partial conclusion one notices that it comes a decisive feature which makes this formalism relevant for analyzing the linearly coupled systems. It could be possible to determine the coupling strength as well as the distance of the beam from the linear difference coupling resonance whitout scanning in tune the machine. Indeed computing $Q_{1}, Q_{2}$ and $r$ at a certain point gives $\left|C_{-}\right|$and $\Delta_{-/+}$. Consequently the strength of the coupling may be measured without jeopardizing the beam by crossing some dangerous resonances.

Another interesting property of this formalism appears when the case of coupling resonances $\left(r \neq \frac{\sqrt{2}}{2}\right)$ is not considered. Merging the two equations of 4.6 gives:

$$
\left|C_{-}\right|=\frac{2 r \sqrt{1-r^{2}}}{2 r^{2}-1}\left|Q_{x}^{0}-Q_{y}^{0}-p\right|
$$

This relation points out that for a certain unperturbed optics the coupling strength is only $r$ dependent. According to the equation $4.4\left|C_{-}\right|$is arclength dependent so this formula shows that $r$ also depends on the arclength. Consequently if $\left|C_{-}\right|$changes after crossing a new element which introduces more coupling $r$ will also change. This reasoning also indicates that $r$ is constant between two elements introducing coupling: a change of $r$ indicates the position of the elements which introduce coupling.

The next graph illustrates the behaviour of $\left|C_{-}\right|$when $r$ varies for a constant unperturbed optics of $\left|Q_{x}^{0}-Q_{y}^{0}-p\right|=0.1$ : 


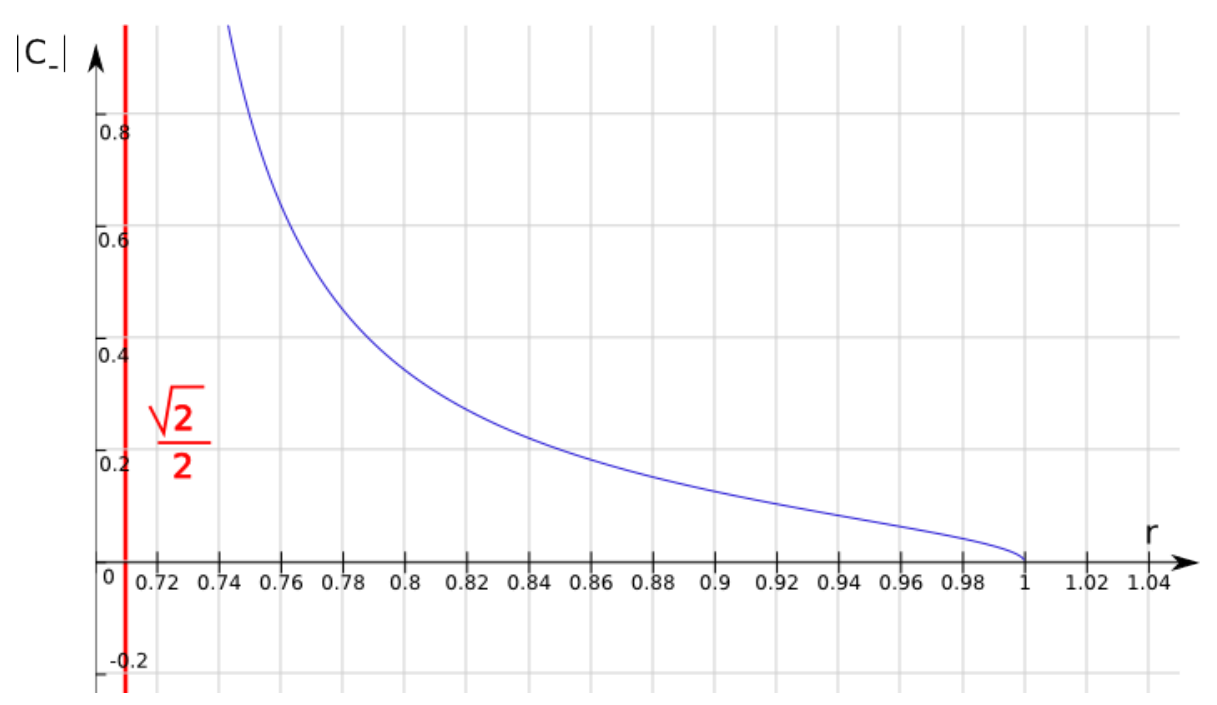

Figure 4.4: The width of the eigentune stopband due to a weak linear difference resonance introduced by a weak and linear coupling. It is a function of the coupling parameter $r$ and is plotted for $\left|Q_{x}^{0}-Q_{y}^{0}-p\right|=0.1 . \frac{\sqrt{2}}{2}$ is the right-limit for $r$.

The values near to 1 but superior are not considered because they represent an unstable case: the weak linear sum resonances.

One deduces from this graph that, at a constant unperturbed optics, the closer to $\frac{\sqrt{2}}{2} r$ is the larger the stopband is i.e. the stronger the coupling is.

As a conclusion of the interpretation of the coupling parameters, it has been shown that the coupling parameter $r$ is related to the coupling strength and to the type of resonance which mainly threatens the beams. In addition $r>1$ corresponds to a case where the system is closer to a linear sum resonance than a linear difference resonance. $\frac{\sqrt{2}}{2}<r<1$ describes the opposite situation. $r<\frac{\sqrt{2}}{2}$ has been excluded by the convention of notations: $Q_{1}$ considered as the perturbed $Q_{x}^{0}$ and $Q_{2}$ the perturbed $Q_{y}^{0}$. Then it has been shown that the lower $r$ is the stronger the coupling is. Its relation with the Hamiltonian pertubation parameters $\left|C_{-}\right|$and $\Delta_{-}$assumes a weak and linear coupling. Moreover the derived equations 4.6 and 4.8 are only valid for the linear difference resonances. Finally the matrix $\mathcal{C}$ is correlated to the phase of $C_{-}$and does not have a direct physical meaning. 


\section{EDWARDS AND TENG'S PARAMETRIZATION IMPLE- MENTED INTO ZGOUBI}

\subsection{EDWARDS and TENG's parametrization in ForTRAN 77}

The formalism previously introduced has been translated into a ForTRAN 77 executable called ETparam. It is compiled from ForTRAN 77 files which are arranged in two main parts.

On one hand a subroutine called tunesc.f has been written for calculating the eigentunes and the eigenvectors of the output matrix given by the regular ZGOUBI: the one-turn mapping. This matrix is stored into an input file called transfertM.dat. Then twiss.f computes the coupling parameters and the generalized Twiss' parameters which, combined with the eigentunes, yields the Hamiltonian perturbation theory parameters to be determined in hamilt.f. An optional subroutine named propag.f can be called if the coupling parameters and the generalized Twiss' parameters need to be transported along the system. Finally all these parameters are written by extrac.f into an output file ETparam.res.

On another hand mathematical libraries from online databases have been downloaded in order to provide to tunesc.f some mathematical subroutines used for the diagonalization, matrix inversion, computation of determinant, etc. The architecture of this program is summarized by the following graph:

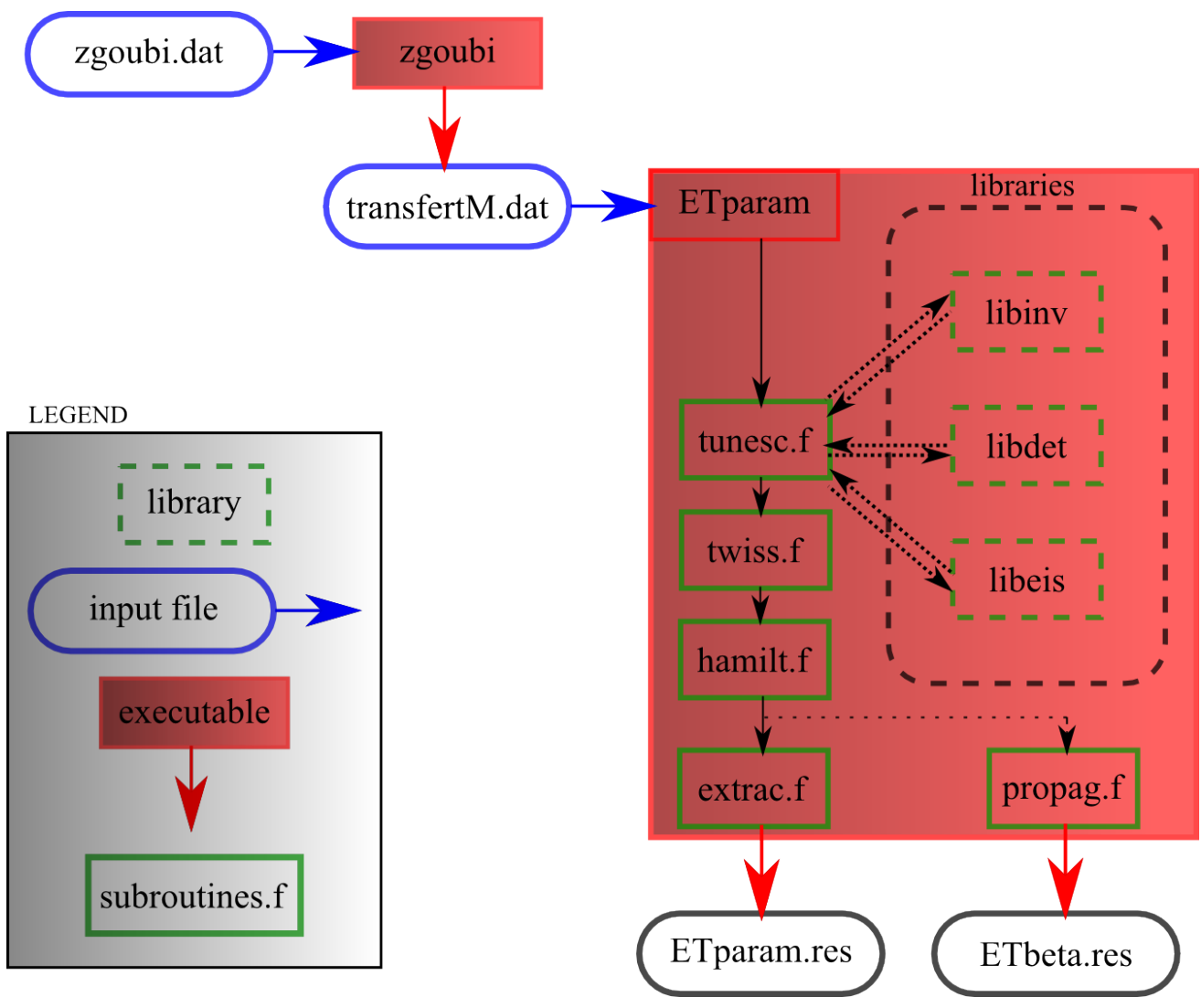

Figure 5.1: Architecture of the code which uses the EDWARDS and TenG's parametrization for computing the eigentunes, the coupling parameters and the generalized Twiss' parameters.

It is now possible to compute the eigentunes and to compare them with the values previously calculated (see table 2.1) by the version of ZGOUBI which does not use the EDWARDS and TENG's parametrization. 


\subsection{Eigentunes}

The table 5.1 compares the betatron tunes computed by ZGOUBI plus the EDWARDS and TENG's parametrization for the systems presented in the subsection 2.2. $E T$ refers to the results computed by $Z G O U B I$ plus ETparam. As explained in the subsection $2.3 Q_{y}^{Z G O U B I}$ and $Q_{x}^{Z G O U B I}$ refer to the tunes calculated from the elements of the one-turn mapping by the previous version of $Z G O U B I . Q_{y}^{D F T}$ and $Q_{x}^{D F T}$ refer to the tunes calculated by a discrete FOURIER transform of the 500-turns ray-tracing of a non-centered particle:

\begin{tabular}{|c|c||c|c|c|c|c|c|}
\hline & & $Q_{2}^{E T}$ & $Q_{y}^{D F T}$ & $Q_{y}^{Z G O U B I}$ & $Q_{1}^{E T}$ & $Q_{x}^{D F T}$ & $Q_{x}^{Z G O U B I}$ \\
\hline \hline \multirow{2}{*}{ No Coupling } & system 1 & 0.194001 & 0.193996 & 0.194001 & 0.163407 & 0.163407 & 0.163407 \\
\cline { 2 - 7 } & system 3 & 0.970006 & 0.970017 & 0.970006 & 0.817035 & 0.817038 & 0.817035 \\
\hline \multirow{2}{*}{ Coupling } & system 2 & 0.182174 & 0.182168 & 0.173906 & 0.134794 & 0.134782 & 0.144466 \\
\cline { 2 - 8 } & system 4 & 0.960235 & 0.960226 & 0.956709 & 0.800959 & 0.800959 & 0.801919 \\
\hline
\end{tabular}

Table 5.1: Comparison of the fractional part of the horizontal betatron tune $Q_{x}$ and the vertical one $Q_{y}$. They have been computed by both $Z G O U B I$ without/with $\left(Q_{i}^{Z G O U B I} / Q_{i}^{E T}\right)$ the EDwARDS and TENG's parametrization and a discrete FouRIER transform $\left(Q_{i}^{D F T}\right)$ of a 500-turns ray-tracing of a non-centered particle.

Corrections have been carried out to the $Z G O U B I$ analyzing process. According to the correspondence between the $Q_{i}^{D F T}$ and the $Q_{i}^{Z G O U B I C}$ the discrepancies have been removed in the systems where the coupling is present.

The next step is to compute the other parameters: $Q_{x}^{0}, Q_{y}^{0},\left|C_{-}\right|, \Delta_{-}$and $\Delta_{+}$. It has been demonstrated that for the coupled system it is possible to compute them from the coupled one-turn mapping via mathematical processes. In order to check the validity of their formulae a tune scan program needs to be developed.

\subsection{Tune scan and GNUPLOT scripts}

A routine has been written in ForTRAN 77 and is called scan.f. It makes the strength of all the defocusing quadrupoles vary in order to scan a range of tunes and to observe the tune split due to the coupling.

In accordance with the coding rules of $Z G O U B I$ the input file extension of the scan program is dat. It contains the scan parameters: starting point, ending point and number of points $\left(N_{\text {points }}\right)$. For each value of the quadrupole strength $K_{Q U A D}$ a new $Z G O U B I$ input file zgoubi.dat is generated. Then ZGOUBI and ETparam are conjointly called in order to compute the eigentunes, the coupling parameters, the generalized TwISs' parameters and the perturbation Hamiltonian theory parameters at each passage into the loop. These values are finally stored into the file fit-scan.in which is the input file of the analyzing process. The tune scan is plotted with GNUPLOT 4.6 by using the script scan.plot. Then the results are fitted by fit.plot with an accuracy of $10^{-8}$. It means that the fit is considered to have converged when the sum of squared residuals changes between two iteration steps by a factor less than $10^{-8}$. The fit parameters are stored into the file fit-scan.out and are used for locating the position of the resonance and its eigentune split $\Delta Q_{\min }$. As it has been explained on the figure 4.2 this value should be theoretically equal to the coupling strength $\left|C_{-}\right|^{E T}$ computed with the EDWARDS and TENG's parametrization.

Nevertheless some discrepancies have been observed and will be presented in the subsection 4.2 . The relative error $\frac{\left|\Delta Q_{\min }-\right| C_{-}||}{\Delta Q_{\min }}$ is plotted and fitted by the script error.plot. These fit parameters are extracted into fit-error.out.

The architecture of the tune scan program is given by the following scheme: 


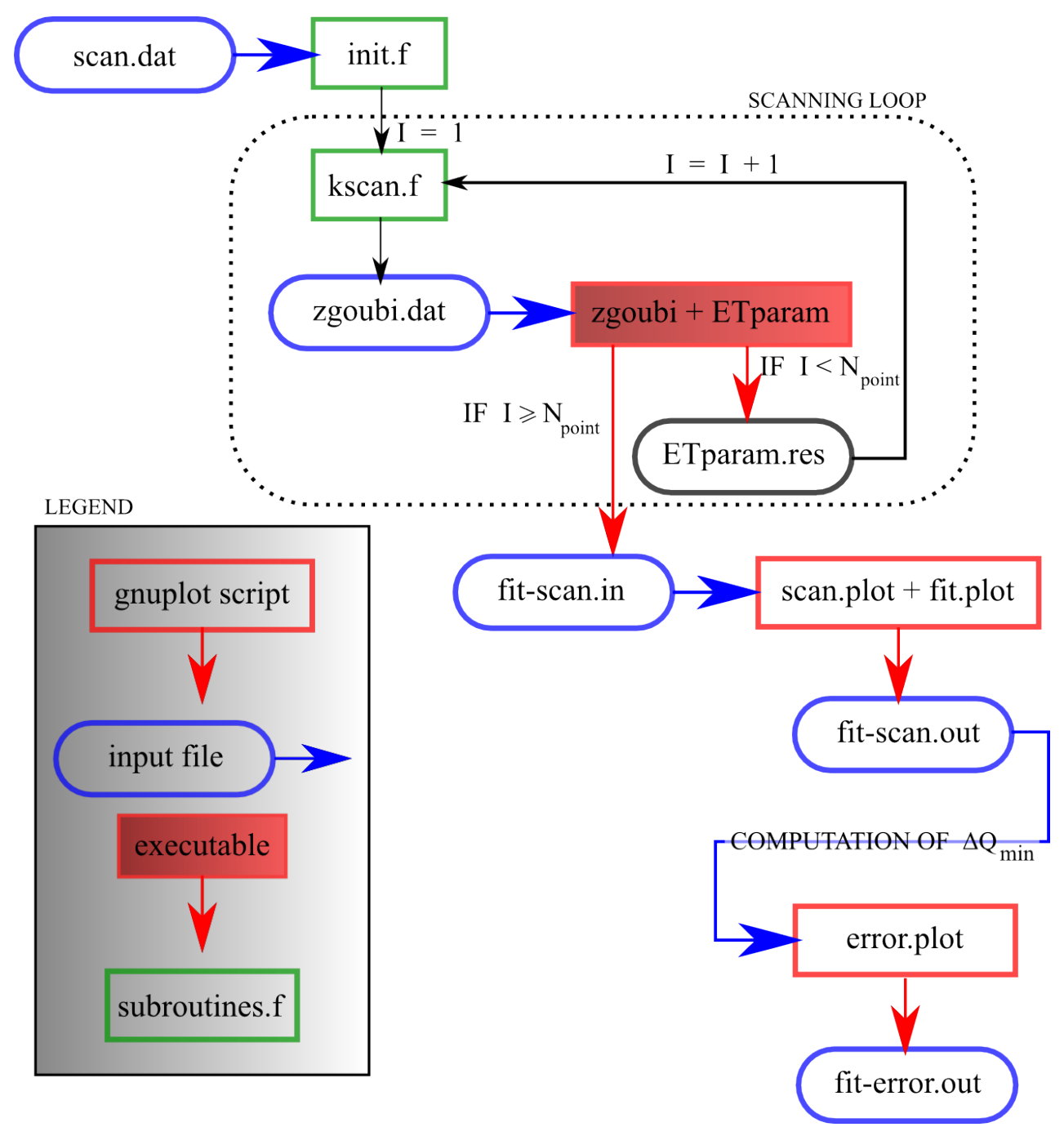

Figure 5.2: Architecture of the code which is used for scanning a system in eigentunes, coupling parameters, generalized Twiss' parameters and perturbation Hamiltonian theory parameters.

\subsection{Tune scan: eigentunes and unperturbed tunes}

The next tests of the benchmarking requires a tune scan for both pair of systems. The case where $r>1$ will not be studied because the Hamiltonian perturbation theory parameters has only been developed for the linear difference resonance.

Tune scans have been launched for both a coupled system with fractional tunes superior to 0.5 (system 3) and one inferior to 0.5 (system 4). For both of them one can see the eigentunes split predicted in the subsection 4.2 . Only the results of the system 4 will be presented in the following graphs. Indeed the results and conclusion concerning the system 2 are similar. 


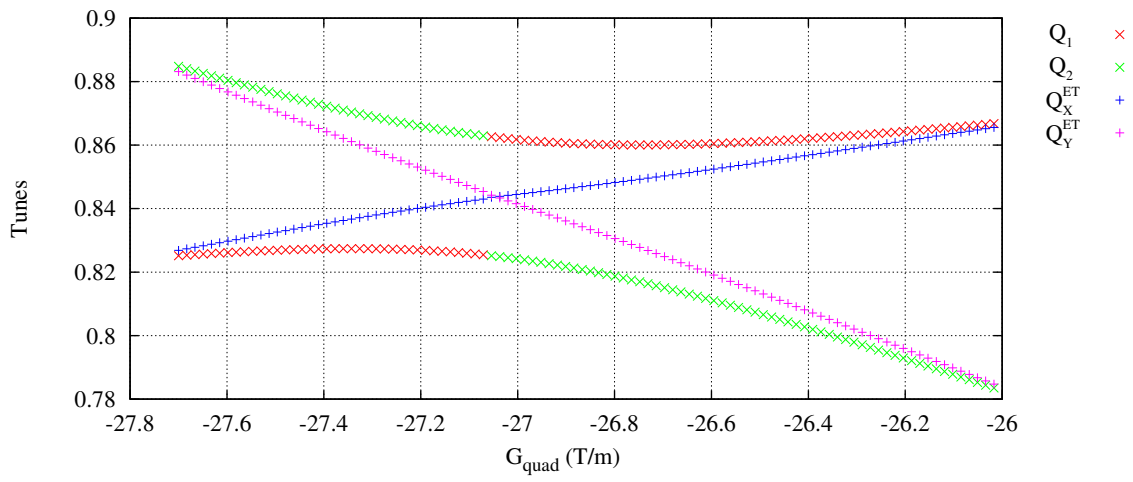

Figure 5.3: Tune scan of the system 4. Both eigentunes $\left(Q_{1}\right.$ and $\left.Q_{2}\right)$ and unperturbed tunes $\left(Q_{x}^{E T}\right.$ and $Q_{y}^{E T}$ ) have been computed for a quadrupole strength range from $-27.7 \mathrm{~T} / \mathrm{m}$ to $-26 \mathrm{~T} / \mathrm{m}$.

In addition the unperturbed tunes $Q_{x}^{E T}$ and $Q_{y}^{E T}$ computed with the EDwards and TEnG's parametrization are the asymptotes of the eigentunes as it has been shown in the figure 4.2 . Consequently one can think that the reconstruction of the unperturbed tunes from a coupled system is correct. Nevertheless it would be better to corroborate this conclusion by plotting both the reconstructed unperturbed tunes $Q_{x}^{E T}$ and $Q_{y}^{E T}$ and the tunes $Q_{x}^{0}$ and $Q_{y}^{0}$ of the equivalent uncoupled systems.

These tunes have been computed for the systems 3 and 4 as well as for their uncoupled counterparts, that are the system 1 and system 2, respectively. It has also been plotted the value of the coupling parameter $r$ in order to know the position of the system compared to the linear difference resonance. It is important to notice that the reconstruction of the unperturbed tunes from a coupled system has only been proposed when $\frac{\sqrt{2}}{2}<r<1$.

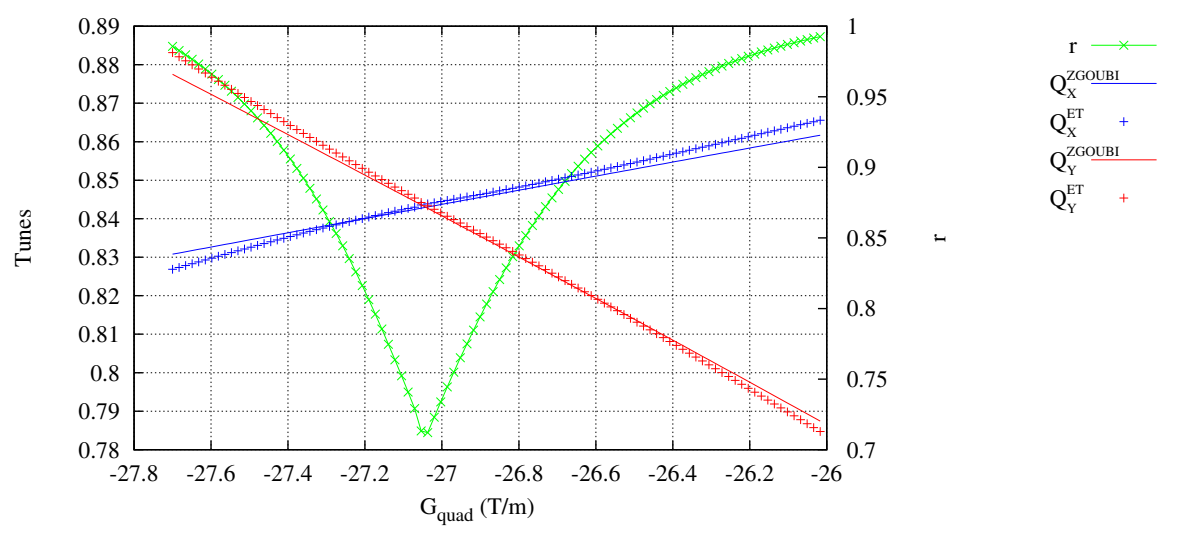

Figure 5.4: Unperturbed tunes computed both for the system 2 (without coupling) and the system 4 (with coupling) during a tune scan for a quadrupole strength varying from $-27.7 \mathrm{~T} / \mathrm{m}$ to $-26 \mathrm{~T} / \mathrm{m}$. $Q_{x}^{0}$ and $Q_{y}^{0}$ are the tunes computed for the system 2 with ZGOUBI. $Q_{x}^{E T}$ and $Q_{y}^{E T}$ are the unperturbed tunes computed for the system 4 with the EDWARDS and TENG's parametrization.

Considering this graph confirms the first conclusion about the accuracy of the reconstructed unperturbed tunes $Q_{x}^{E T}$ and $Q_{y}^{E T}$. One notices small discrepancies of roughly $3 \%$ when the system are almost uncoupled. As the unperturbed tunes are a function of the coupling strength an inaccurate 
computation of it could be source of errors. More details will be given in the subsection 5.5 . Even if the discrepancies are low it is recommended in this case to consider directly $Q_{x}^{0}=Q_{1}$ and $Q_{y}^{0}=Q_{2}$. Indeed the eigenvalues of the one-turn mapping for an uncoupled system are its unperturbed tunes.

\subsection{Hamiltonian perturbation theory parameters}

\subsubsection{Distances from the linear resonances}

$\Delta_{-}$and $\Delta_{+}$have been computed for each system with both ZGOUBI and the EDWARDS and TENG's parametrization. It will allow to compare the ones computed by the regular part of $Z G O U B I$ and the ones computed with the reconstructed unperturbed tunes owing to the EDWARDS and TENG's parametrization and Hamiltonian perturbation theory. According to the following graphs the two ways of computing are in good agreement because the discrepancies are almost not distinguishable. Only the results of the system 4 will be presented with the following graphs. Indeed the results and conclusion concerning the system 2 are similar:

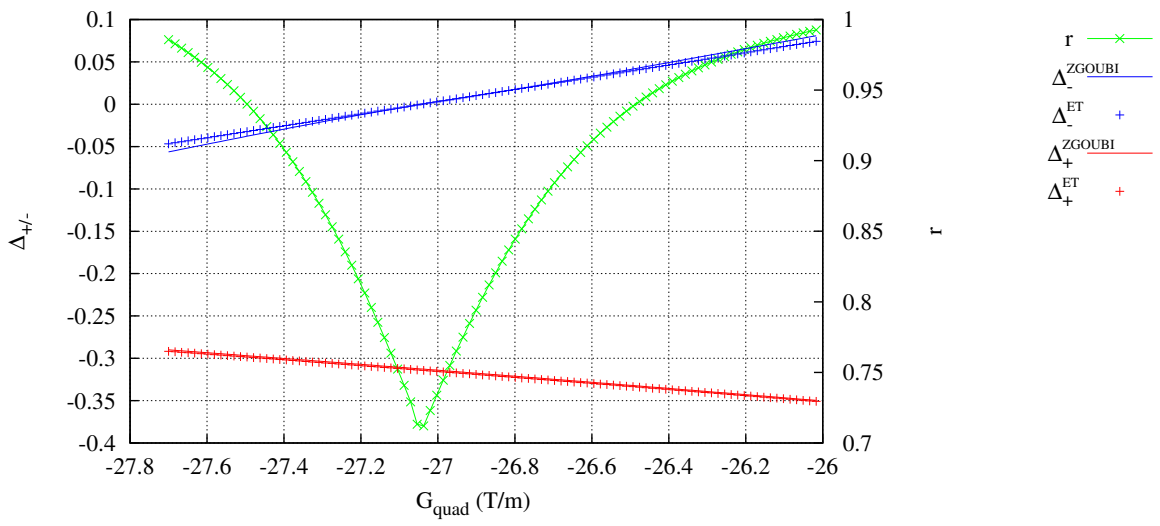

Figure 5.5: Computation of the distance from a linear difference resonance $\left(\Delta_{-}\right)$and a linear sum resonance $\left(\Delta_{+}\right)$during the tune scan both for the system 4 (coupled) and the system 2 (uncoupled). It has been done on one hand by ZGOUBI and on another hand by EDWARD and TENG's parametrization. The systems have been scanned for a quadrupole strength varying from $-27.7 T / m$ to $-26 T / m$. The coupling parameter $r$ is also plotted in order to quantify the coupling.

\subsubsection{Coupling strength of the linear difference resonances}

The coupling strength of a linear difference resonance can be computed by three different ways:

1. Edwards and Teng's parametrization using the equation 4.6 .

2. Thin lens approximation using the definition of $\left|C_{-}\right|$(see equation 4.4).

3. Width of the eigentune stopband during a tune scan (see figure 4.2).

First it has been shown by the formula 4.6 that it can be calculated from the eigentunes and the coupling parameter $r$. It has been done for both the system 2 and 4 but only the results of the system 4 will be presented by the following graphs because the results and conclusion concerning the system 2 are similar. The next graph represents the coupling strength as well as other relevant parameters computed by the EDWARDS and TENG's parametrization: 


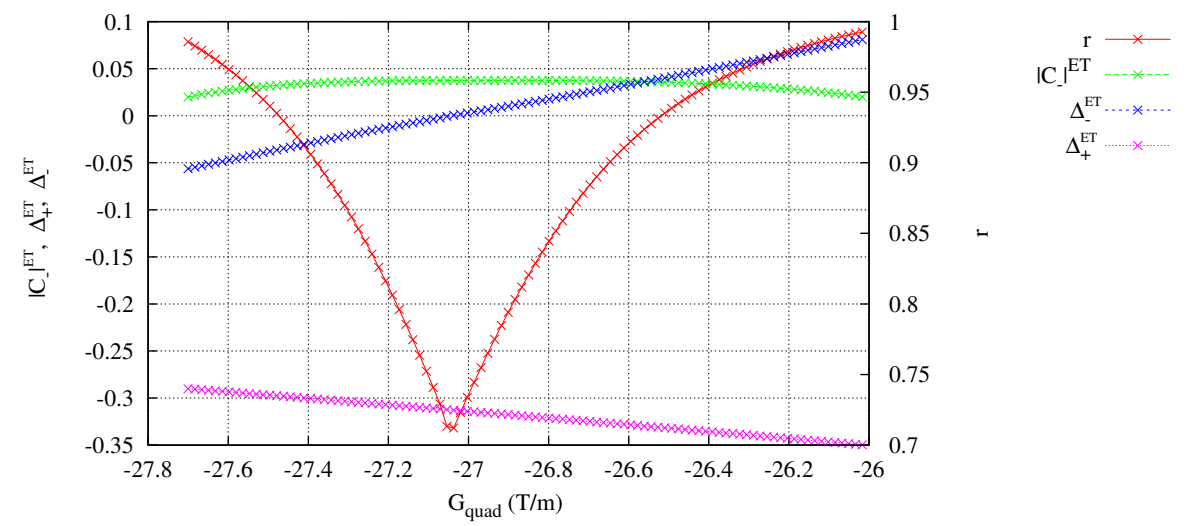

Figure 5.6: Hamiltonian perturbation theory parameters for a tune scan of the system 4 . The coupling parameter $r$, coupling strength $\left|C_{-}\right|^{E T}$ and the tune distances from a linear resonance $\Delta_{+/-}^{E T}$ have been computed for a quadrupole strength range from $-27.7 \mathrm{~T} / \mathrm{m}$ to $-26 \mathrm{~T} / \mathrm{m}$.

As expected the coupling strength is about constant in the vicinity of $\Delta_{-}$whatever the scanning defocusing quadrupole strength is. Indeed the coupling strength should not change if no more coupling is introduced. Nevertheless one can notice that $\left|C_{-}\right|^{E T}$ tends toward zero when the distance in tune from the linear difference resonance increases. It would be better to compare $\left|C_{-}\right|^{E T}$ with values computed by another method.

Secondly one can also compute the coupling strength by assuming the skew quadrupole thin enough such as:

$$
\begin{cases}k_{s}(s)=0.2 m^{-2} & \text { for } s=s_{\text {skew quad }} \\ k_{s}(s)=0 m^{-2} & \text { for } s \neq s_{\text {skew quad }}\end{cases}
$$

So the equation 4.4 becomes:

$$
\left|C_{-}\right|^{\text {thin lens }}=\frac{k_{s} l_{\text {skew }}}{2 \pi} \sqrt{\beta_{x}\left(s_{\text {skew quad }}\right) \beta_{y}\left(s_{\text {skew quad }}\right)}
$$

As $Z G O U B I$ can give the unperturbed $\beta$-values in the skew quadrupole it is possible to compare the coupling strength both computed by this thin lens approximation and the first method. It has been done for some points in the tune scan because it was not so convenient to perform. It only aimed at confirming quickly the order of magnitude given by the first method.

Although the order of magnitude is the same it arises that there are discrepancies between these two methods varying from $5 \%$ to $30 \%$ such as the further away from the resonance the system is the bigger the discrepancy is. These two methods are based on the Hamiltonian perturbation theory so it is not possible to conclude about the pertinence of this model. This second method only shows that the coupling strength computed by ZGOUBI plus the EDWARDS and TENG's parametrization seems to be roughly in agreement around the resonance. Nevertheless it would be better to corroborate this conclusion by computing the coupling strength with a third method not based on an analytical formula.

Finally the coupling strength can also be measured directly from a tune scan. As it has been presented by the figure 4.2 that the coupling strength is also the width of the eigentune stopband. It is interesting to notice that this way of measuring the coupling strength is used experimentally.

Determining the width of the eigentunes stopband from a scan requires to, first, find the location of the resonance, then, to know the eigentunes corresponding this resonant quadrupole strength. Fitting the unperturbed tunes with a line yields the location of the resonance to be accurately determined at 
$k=k_{r e s}$. Then fitting the eigentunes with a second degree polynomial allows to compute them at the resonance and so to calculate the eigentune stopband width:

$$
\Delta Q_{\text {min }}=\left|Q_{1}\left(k_{r e s}\right)-Q_{2}\left(k_{r e s}\right)\right|
$$

The process applied to the scan of the system 4 in the figure 5.7 yields $\Delta Q_{\min }$ and $k_{\text {res }}$ to be computed: $\Delta Q_{\min }=0.039057$ and $k_{\text {res }}=-27.025 \mathrm{~m}^{-2}$. It allows to compare this value with the ones computed by the two first methods. Their relative errors are plotted on the figure 5.8 .

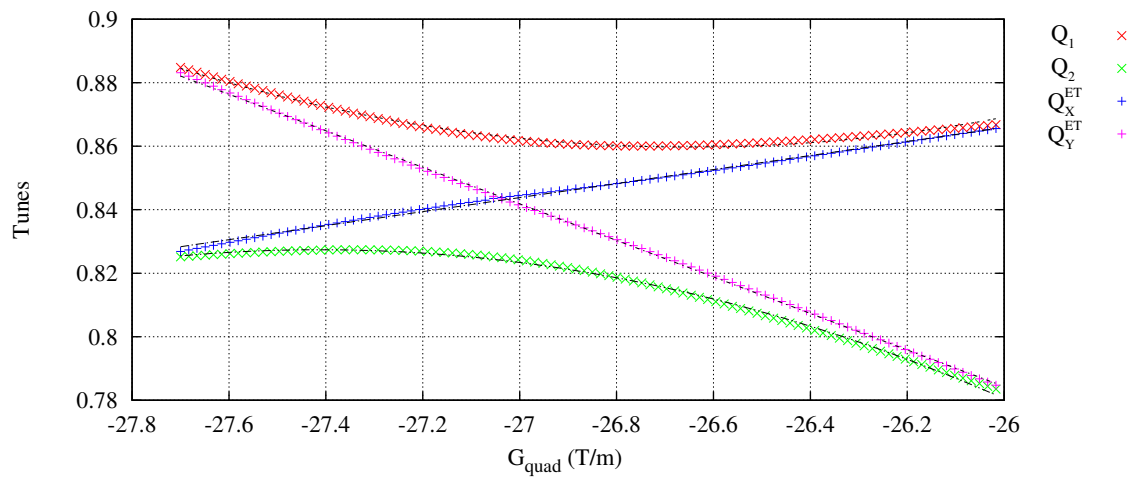

Figure 5.7: Tune scan of the system 4. Both eigentunes $\left(Q_{1}\right.$ and $\left.Q_{2}\right)$ and unperturbed tunes $\left(Q_{x}^{E T}\right.$ and $Q_{y}^{E T}$ ) have been computed for a quadrupole strength range from $-27.7 \mathrm{~T} / \mathrm{m}$ to $-26 \mathrm{~T} / \mathrm{m}$. The black lines are the fit of each tunes. The unperturbed tunes have been linearly fitted and the eigentunes have been fitted with a second degree polynomial.

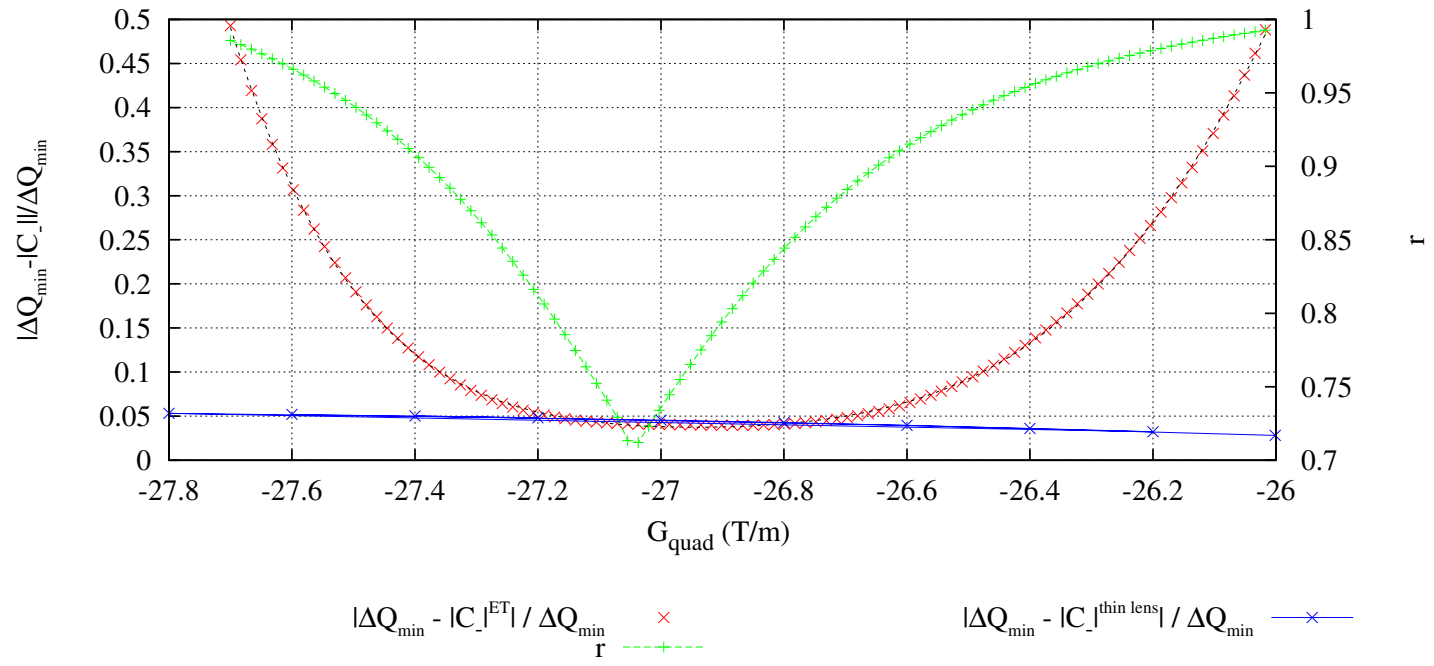

Figure 5.8: Relative error of the coupling strength computed for each value of a tune scan made for the system 4 . The black line is its fit: a $6^{\text {th }}$-degree polynomial. The coupling parameter $r$ has also been plotted as a indicator of the tune position relative to the linear difference resonance. It has been computed for a quadrupole strength range from $-27.7 \mathrm{~T} / \mathrm{m}$ to $-26 \mathrm{~T} / \mathrm{m}$. 
As observed with the two first methods the coupling strength computed with EDWARDS and TENG's parametrization seems to be in accordance with $\Delta Q_{\min }$ around the resonance $(\lesssim 5 \%)$ but it diverges when the system moves away from the resonance. In addition to corroborate this previous conclusion the figure 5.8 also shows that the relative error behaves like a $6^{\text {th }}$-degree polynomial.

This effect needs to be investigated. It may be due to the betatron function dependence of $\left|C_{-}\right|$ (see equation 5.2). Indeed changing the defocusing quadrupole strength will change the unperturbed betatron functions and may have a non-neglegible effect on $\left|C_{-}\right|$. The blue curve of the figure 5.8 is

the relative error between $\Delta Q_{\text {min }}$ and $\left|C_{-}\right|^{\text {thin lens }}$. It shows that the computation of $\left|C_{-}\right|^{\text {thin tens }}$ is few affected by the change of the defocusing quadrupole strength so the variations of $\left|C_{-}\right|^{E T}$ are not due to the betatron function dependence of $\left|C_{-}\right|$. The source of this error has not been found in this study and still have to be investigated.

\subsection{Propagation of the generalized TwISs' parameters and the coupling parameter $r$ along the Alternating Gradient Synchrotron}

The transport of the coupling parameters and the generalized TwISS' parameters will be presented for the Alternating Gradient Synchrotron. Indeed this study requires to compare the values computed by the EDWARDS and TENG's parametrization to the ones given by a software able to deal with coupled systems.

But first the AGS will be briefly described.

\subsubsection{Features of the Alternating Gradient Synchrotron}

The AGS is a 804 meters circumference synchrotron. It accelerates polarized protons from 2.3 to $23.8 \mathrm{GeV}$ in 400 milliseconds. Then it prepairs the beam for injection into the collider RHIC. Its whole cycle lasts roughly three seconds.

It is composed of 240 combined functions dipoles. These magnets are qualified of combined functions because they bend and focus at the same time the beam. There are three different types of dipoles:

1. Type A: 90 inches $(228.6 \mathrm{~cm})$ long and gap opened toward the exterior of the ring.

2. Type B: 75 inches $(190.5 \mathrm{~cm})$ long and gap opened toward the exterior of the ring.

3. Type C: 90 inches long and gap opened toward the interior of the ring.

The special feature of the AGS is that it contains two siberian snake magnets? They are used for reducing the proton polarization loss during the acceleration. Additional magnets such as quadrupoles and sextupoles are positioned along the ring in order to adjust the betatron tune and the chromaticity.

\subsubsection{Propagation of the generalized TwIss' parameters}

A model of the AGS is used for testing the validity of the formulae computing the generalized TwISS' parameters along the ring. The settings of the accelerator have been taken for a timing of 162 milliseconds which is located at the beginning of a cycle. The coupling at this timing is stronger. The next graph plots the generalized $\beta$ functions for the first 200 meters:

\footnotetext{
${ }^{7}$ Siberian snake magnets: For additional information see appendix B.2
} 


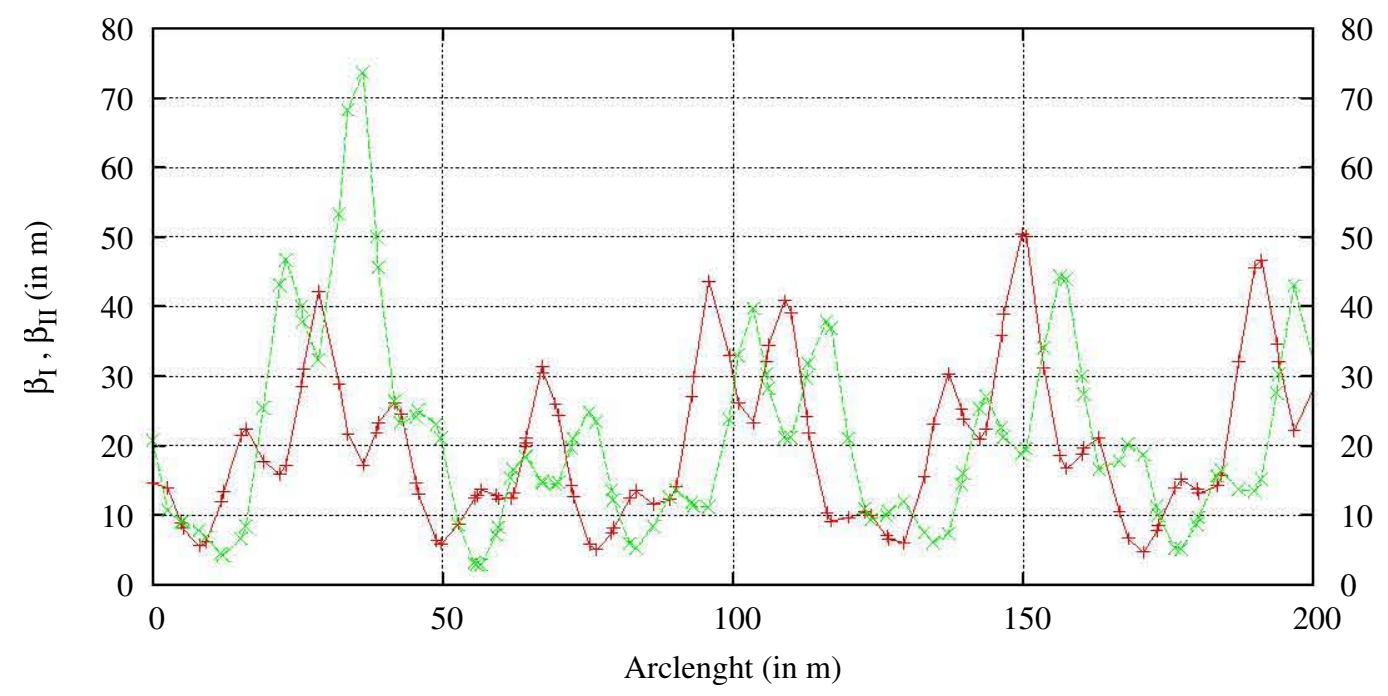

$\beta_{\mathrm{II}}$

Figure 5.9: Generalized $\beta$ functions for the mode I and II along the first 200 meters of the Alternating Gradient Synchrotron computed with ZGOUBI plus the Edwards and Teng's parametrization. These data have computed for a timing of 162 milliseconds.

Each generalized $\beta$ function has been compared to the $M A D X$ simulated ones:
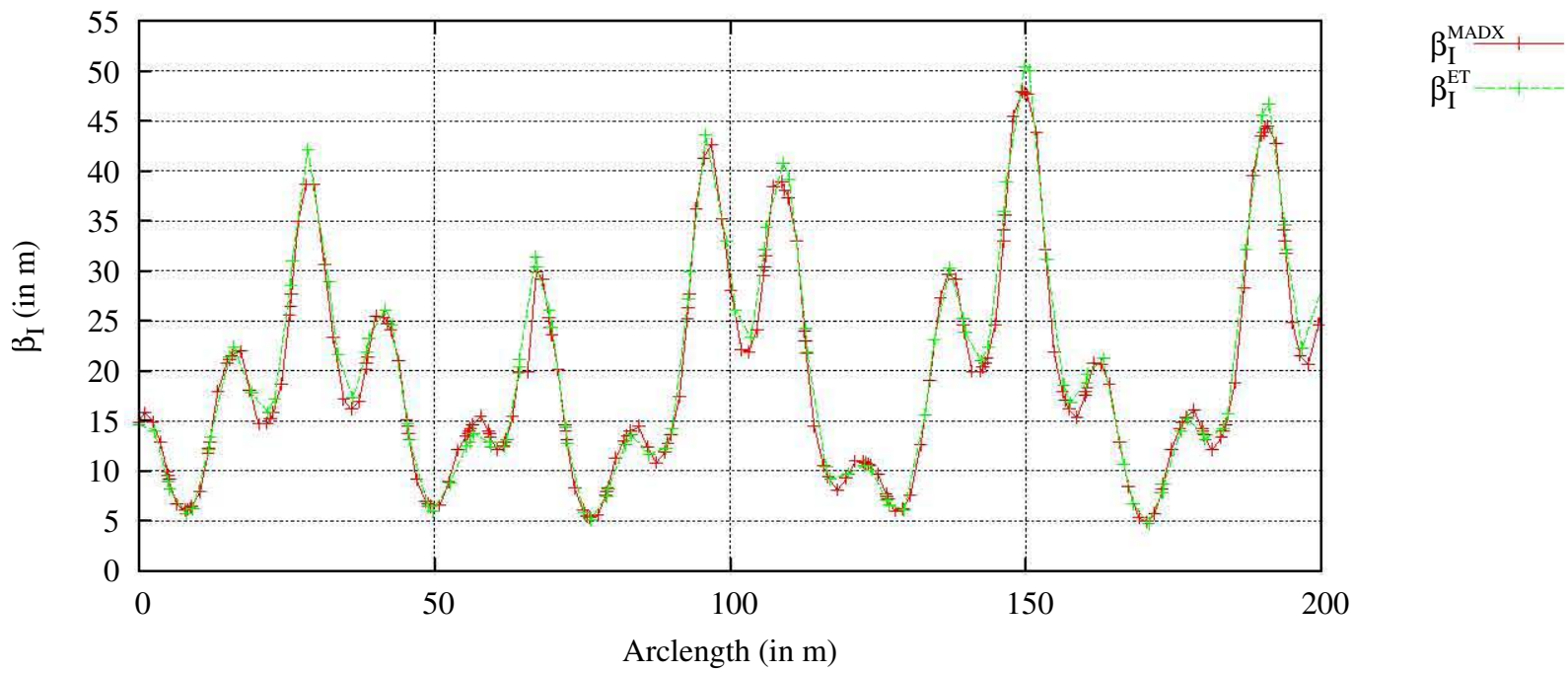

$\beta_{\mathrm{I}}^{\mathrm{ET}}$

Figure 5.10: Generalized $\beta$ functions for the mode I along the first 200 meters of the Alternating Gradient Synchrotron computed both with MADX and ZGOUBI plus the EDWARDs and Teng's parametrization. These data have been computed for a timing of 162 milliseconds. 

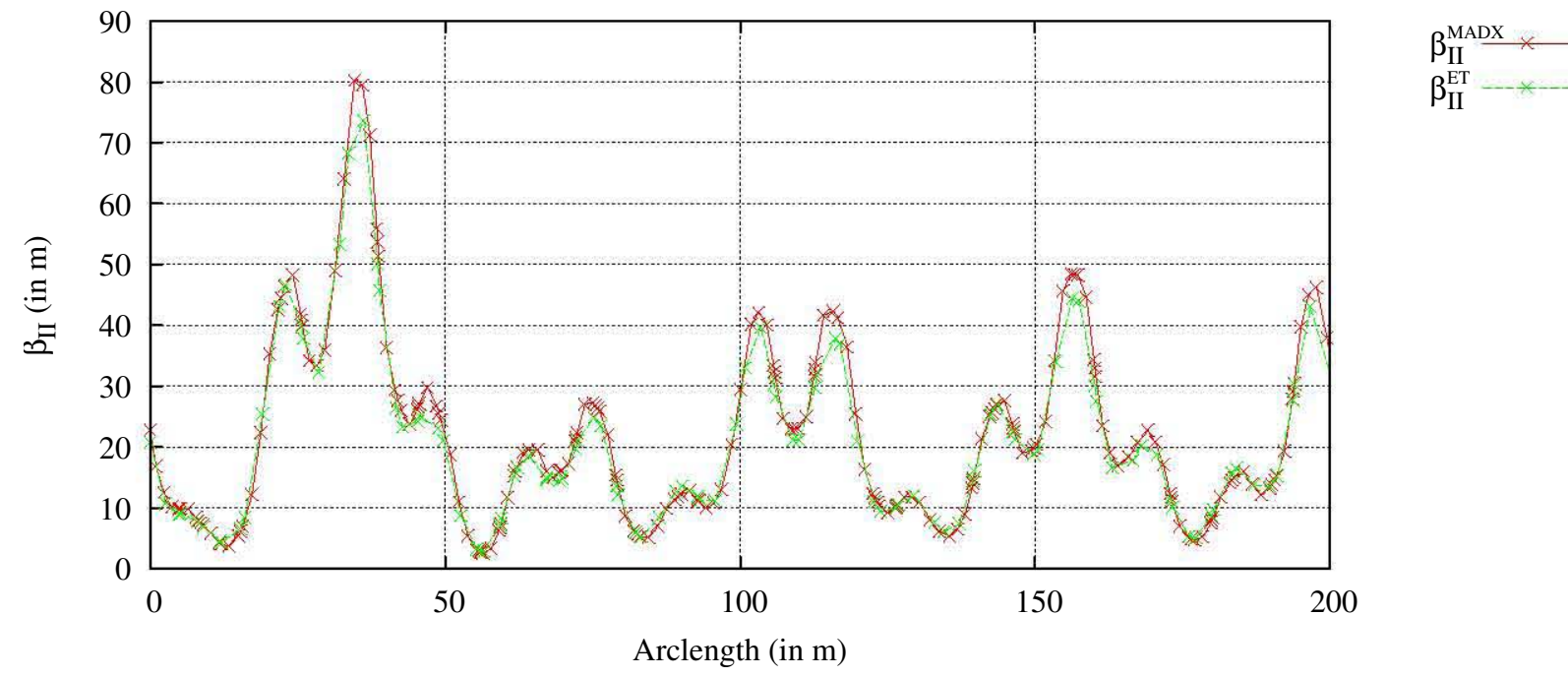

Figure 5.11: Generalized $\beta$ functions for the mode II along the first 200 meters of the Alternating Gradient Synchrotron computed both with MADX and ZGOUBI plus the Edwards and Teng's parametrization. These data have been computed for a timing of 162 milliseconds.

The two softwares are in good agreement. According to the definition of the Twiss' parameters in the subsection 1.2, if the generalized $\beta$-functions are in good agreement thus the generalized $\alpha$ functions and $\gamma$-functions are also in good agreement. Consequently it indicates that the computation of the generalized Twiss' parameters along the ring with the EDWARDS and TENG's parametrization implemented into $Z G O U B I$ is correct.

However one notices little discrepancies at the peaks of the generalized $\beta$-functions. ZGOUBI and $M A D X$ are both using the EDWARDS and TENG's parametrization so these differences may come from the beam/particle simulations prior to the analysis of the coupled motion. ZGOUBI is a numerical integrator whereas $M A D X$ is based on a matrix representation of the accelerator. The spatial resolution of ZGOUBI is only limited by the integration step which is defined by the user. On contrary $M A D X$ makes its computations element by element. Increasing the accuracy of its calculus is synonym to slicing the elements of the machine into smaller elements.

The generalized $\beta$ functions for the mode I and II along the first 200 meters have also been computed for different timings of the acceleration process. It will help to have a global view of the generalized $\beta$ functions evolution during the whole acceleration process: 


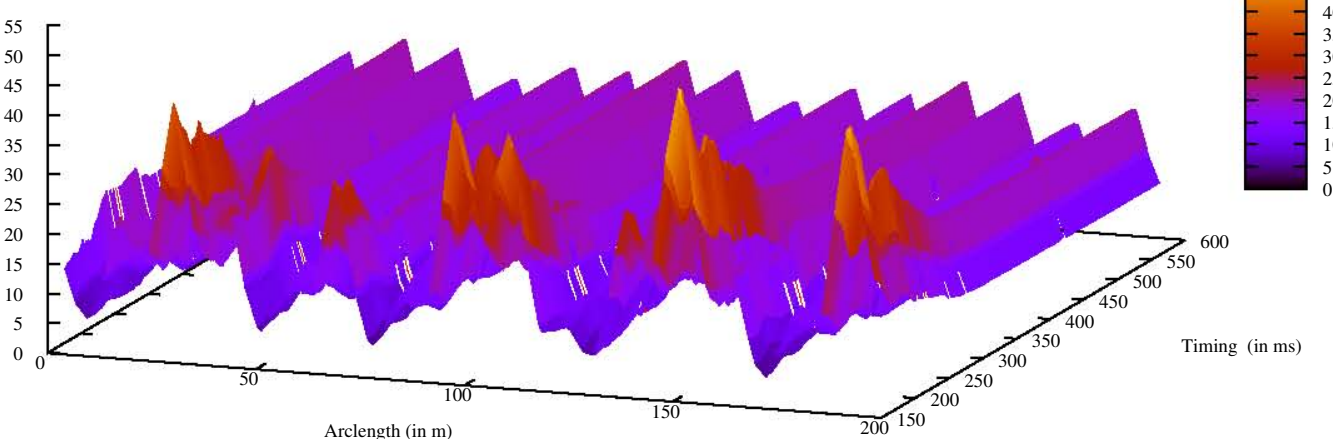

Figure 5.12: Generalized $\beta$ function for the mode I along the first 200 meters of the Alternating Gradient Synchrotron computed with ZGOUBI plus the Edwards and Teng's parametrization. These data have computed along the acceleration process, from 160 to 582 milliseconds.

One notices that the more accelerated the beam is the smaller the generalized $\beta$ functions are. It was expected because the effect of the siberian snake on the beam is inversely proportional to the beam momentum so the less accelerated the beam is the more perturbed it is.

\subsubsection{Propagation of the coupling parameter $r$}

As for the generalized Twiss' parameters the coupling parameter $r$ has been transported along the ring for the same AGS settings. The result is displayed by the following graph:

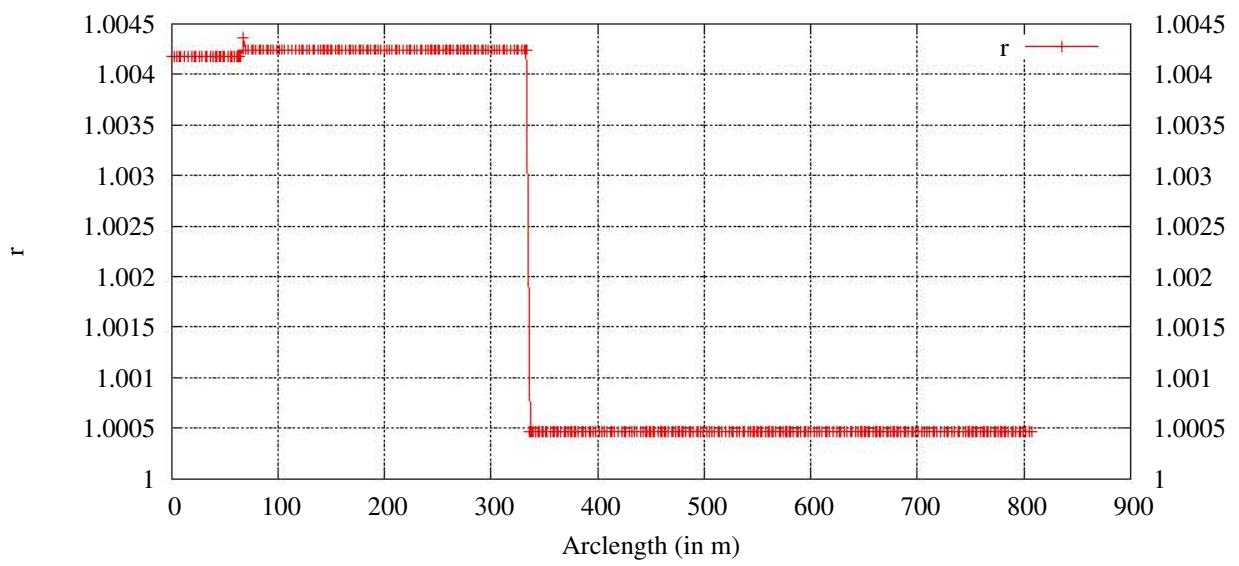

Figure 5.13: Coupling parameter $r$ along the Alternating Gradient Synchrotron computed with $Z G O U B I$ plus the EDWARDS and TENG's parametrization. These data are computed for a timing of 162 milliseconds. 
One notices $r$-jumps as predicted by the theory exposed in the subsection 4.3 . It has been shown that $r$ is a constant parameter between two elements introducing coupling but makes jump when the system crosses them. So a change of the coupling parameter $r$ hints the position of the coupling sources. In the AGS model which is used the coupling sources are the siberian snake magnets. They are exactly located at the $r$-jumps displayed in the previous graph.

The order of magnitude of the $r$ numerical variations when the siberian snakes magnets are turnedoff is about $10^{-8}$ (see figure 5.14). These fluctuations are due to the numerical errors. It defined resolution in $r$ for locating the $r$-jump and so the coupling sources.

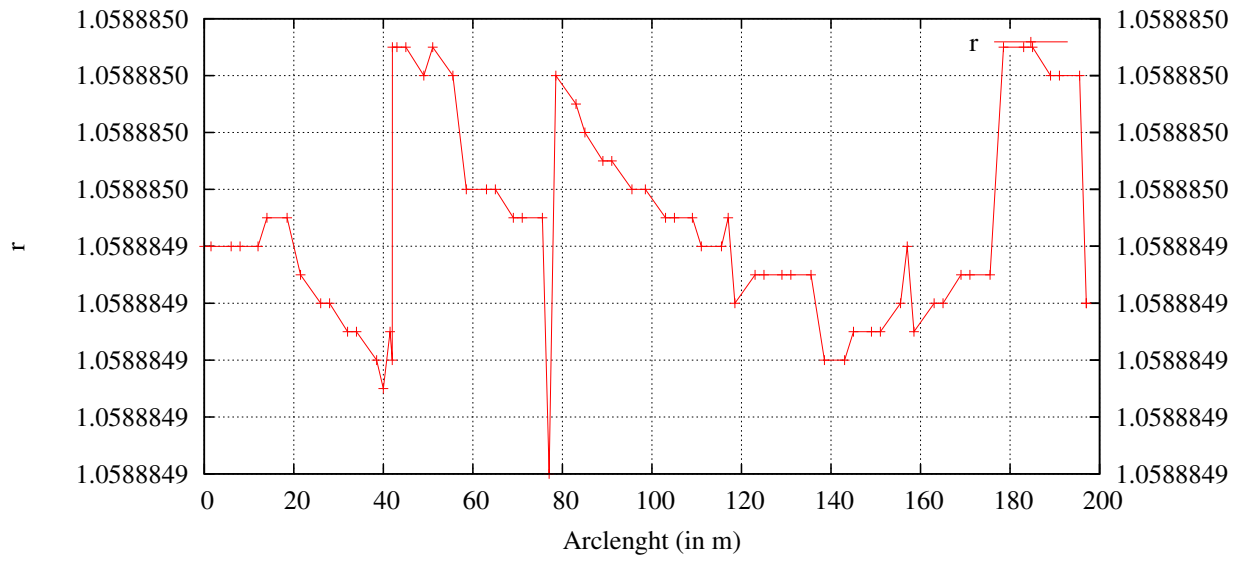

Figure 5.14: Coupling parameter $r$ along the first 200 meters of the Alternating Gradient SynChrotron computed with ZGOUBI plus the Edwards and TenG's parametrization. These data have been computed for a timing of 162 milliseconds and the siberian snake magnets turned-off.

The next graph points out that $r$ is not a periodic function. It has been computed for a particle making 10 turns in the AGS at 162 milliseconds:

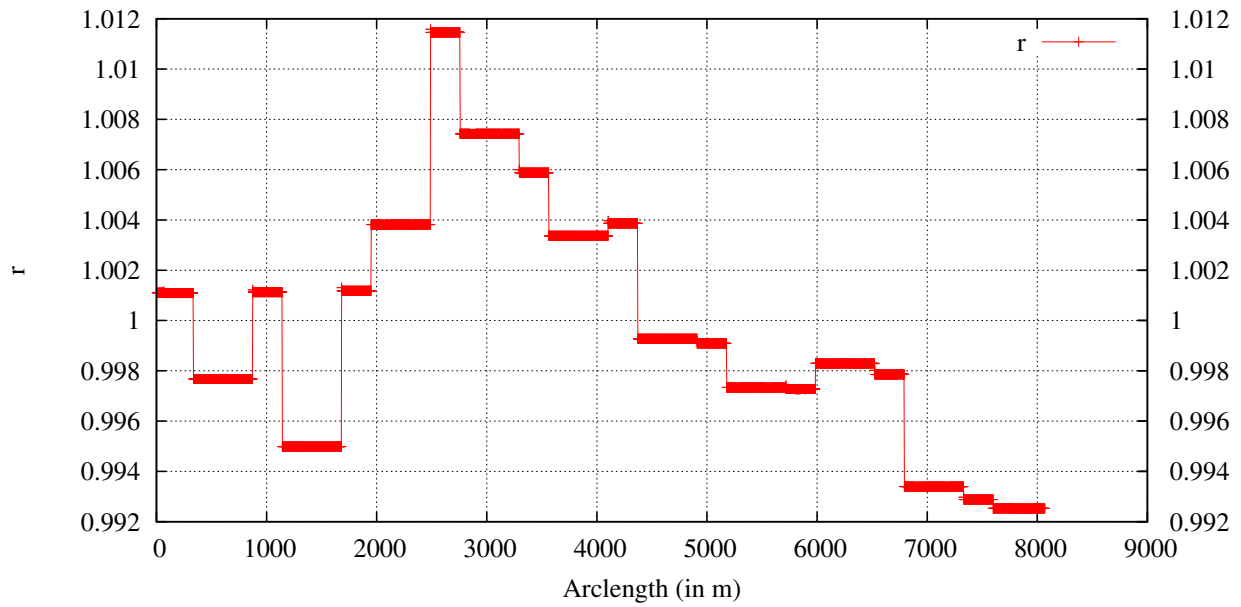

Figure 5.15: Coupling parameter $r$ along the Alternating Gradient Synchrotron computed with ZGOUBI plus the EDWARDS and TENG's parametrization. These data are computed for a timing of 162 milliseconds over 10 turns. 


\section{CONCLUSION \& PROSPECTS}

During this 5-months internship the weak and linear coupling occuring in accelerators was studied. It was noticed owing to $Z G O U B I$ simulations that the uncoupled formalism needs to be generalized for treating with $Z G O U B I$ the one-turn mapping of coupled systems. As a consequence a mathematical formalism treating the coupling was searched in order to implement it into ZGOUBI.

It was found out that the EDWARDS and TENG's parametrization is currently used in several codes dedicated to general purpose accelerator simulations such as $M A D X$. Then one went through the details of this formalism in order to propose a synthesis. It introduced the EDWARDS and TENG's parameters which corresponds, on one hand, to the generalized Twiss' parameters and, on another hand, to the coupling parameters $r$ and $\mathcal{C}$. One developed physical analyses for helping the interpretation of these new parameters and the understanding of future simulations. It required to use both a matrix approach and a perturbation Hamiltonian theory defining new variables such as the coupling strenght $\left|C_{-}\right|$as well as the tune distance from a linear coupling resonance $\Delta_{-/+}$and the unperturbed tunes $Q_{x}^{0}$ and $Q_{y}^{0}$. It has been derived relations between the EDwards and TenG's parameters and the Hamiltonian perturbation ones. In the frame of a weak linear difference resonance it has been shown that the lower $r$ is the stronger the coupling is and the phase of $C_{-}$is correlated to the matrix $\mathcal{C}$ whitout having a direct physical meaning. $r>1$ corresponds to a case where the system is closer to a linear sum resonance than a linear difference resonance. The case described by $\frac{\sqrt{2}}{2}<r<1$ is the opposite situation. $r=1$ correspond to an uncoupled system and $r=\frac{\sqrt{2}}{2}$ a system on the resonance.

New simulations have been handled with the EDWARDS and TENG's parametrization implemented into ZGOUBI for some benchmark systems. First, it came out a better precision of eigentune computations, it was reduced from $10^{-2}$ to $10^{-6}$. Then it was demonstrated that the unperturbed tunes and so the tune distance from the linear coupling resonances are computed with a good accuracy. Nevertheless one found out that the coupling strength established by the Hamiltonian perturbation approach is only valid at the vicinity of the linear difference resonances.

Finally the propagation of the EDWARDS and TENG's parameters along the ring was tested with a model of the Alternating Gradient Synchrotron. The transported generalized Twiss parameters along the ring were in good agreement with the ones computed by $M A D X$. The coupling parameter $r$ was also transported along the ring. First it showed that $r$ is not a periodic function then it exhibited a theoretically predicted phenomenon. $r$ stayed constant in section where no coupling is introduced whereas it made a jump when it crossed a source of coupling. Thus the propagation of the EDwards and TENG's parameters hinted the location of the coupling sources in the AGS. Only two jumps were seen as expected. They refered to the only source of coupling the AGS model used for these simulations: the siberian snake magnets.

Although the location of coupling sources was already known in these simulations this is not the case for the experimental facility. There are some unpredicted and unlocated sources of coupling which need to be understood. The topic of future studies could be the application of the EDWARDS and TENG's formalism to AGS experimental data in order to locate the coupling sources. 


\section{APPENDIX A: FRAMEWORK}

\section{A.1 The Brookhaven National Laboratory}

The Brookhaven National Laboratory (BNL) [10] is a laboratory led by the United States Department of Energy. It is staffed by approximately 3,000 scientists, engineers, technicians, and support personnel, and hosts 4,000 guest investigators every year. Discoveries made at the lab have won five Physcis Nobel prizes and two Chemistry Nobel Prizes. BNL is a world-wide known for its research in :

- Nuclear and high-energy physics

- Physics and chemistry of materials

- Environmental energy research

- Nonproliferation

- Neurosciences \& Medical imaging

- Structural biology

In addition to the high level research in these fields, research groups of the whole world come to the BNL for leading experiments. Indeed, its research facilities make it unique and attractive. The main facilities are the Center for Functional Nanomaterials (CFN) using for nanoscale materials studies, the New York Blue Gene supercomputer which is the world's $5^{\text {th }}$ fastest supercomputer, and its particle accelerators.

\section{A.2 Laboratory's particle accelerators}

The BNL has two main facilities: the National Synchrotron Light Source [11] and the RelaTivistic Heavy Ion Collider complex [12].

The National Synchrotron Light Source (NSLS) is considered as a second generation synchrotron. The experimental set up consists of two electron storage rings: an X-Ray ring and a Vacuum Ultra Violet Ring. They provide an intense focused light spanning the electromagnetic spectrum from the infrared through $\mathrm{x}$-rays used by scientists in many fields for performing experiments unrealizable at their own laboratories. A new synchroton called the NSLS-II is under construction. Using a new state-of-the-art it will be a $3 \mathrm{GeV}$ electron storage ring designed to deliver world-leading intensity and brightness. The NSLS-II X-Ray will be more than 10,000 times brighter than the current ones. These facilities will not be detailed anymore because I did not work on them.

The Relativistic Heavy Ion Collider complex consists of a chain of particle accelerators that prepair the beam for colliding into the Relativistic Heavy Ion Collider (RHIC).

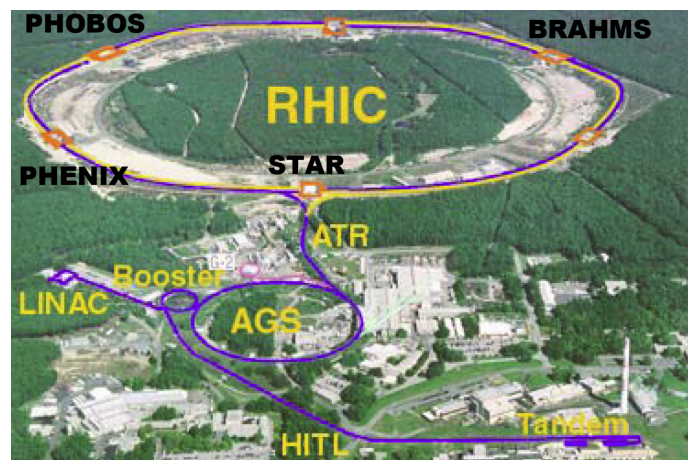

Figure 5.16: Relativistic Heavy Ion Collider complex 
Ion \& Proton sources: A tandem of VAN DE GRAAFF electrostatic accelerators serves as ion source for the RHIC. It uses static electricity to accelerate atoms removing some of their electrons by a $15 \mathrm{MV}$ static electric field. From the Tandem, the bunches of ions enter the TANDEM-TO-Booster beamline, which carries them at about $5 \%$ the speed of light through a vacuum via a magnetic field to the Booster Synchrotron. In addition to heavy ions, some experiments at RHIC need to study the colliding of polarized proton beams. The protons are supplied by a $200-\mathrm{MeV}$ Linac and are directly transferred to the BoosTER.

The Booster Synchrotron: The Booster synchrotron is a circular accelerator added to the facility in 1991. It was designed for allowing the next accelerator -the Alternating Gradient Synchrotron (AGS)- to accelerate and deliver heavy ions up to gold. It provides the ions and the protons more energy by using radiofrequency acceleration. Nowadays the synchrotron feeds the beam into the AGS with particles traveling at about $37 \%$ the speed of light.

Alternating Gradient Synchrotron: As in the booster, the beam is accelerated in the AGS and reaches $99.7 \%$ of the light speed. Moreover a system of alternating gradient focusing is used for permitting particles to be focused in both the horizontal and vertical plane at the same time. When the beam is traveling at top speed in the AGS, it is taken down another beam line called the AGS-To-RHIC (ATR) transfer line. At the end of this line there is a switching magnet which sends the bunches down one of two beam lines. Bunches are directed either left to the clockwise RHIC ring (called blue ring, figure 5.16) or right to travel counter-clockwise in the second RHIC ring (called yellow ring).

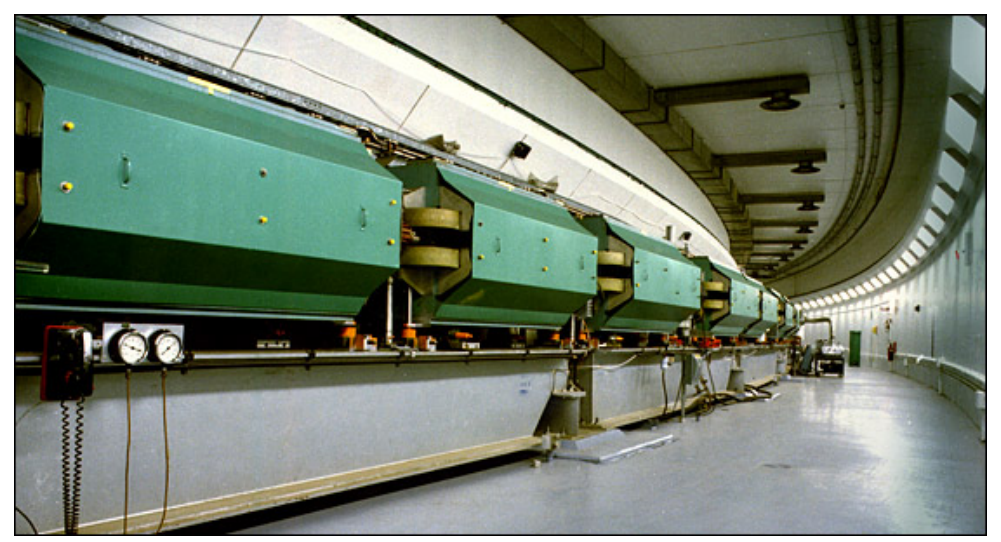

Figure 5.17: Alternating Gradient Synchrotron

Relativistic Heavy Ion Collider: The RHIC is the $2^{\text {nd }}$ most powerful hadron collider, its counter-rotating beams are accelerated along its 2.4 mile ring until $99.995 \%$ the speed of light. Then polarized protons are collided one into the other at as many as six interaction points (orange squares) where its two rings cross.

Two different types of collisions are carried out at RHIC. The particles used are either polarized protons or heavy ions: gold, copper... The collisions of heavy ions produce the study of the primary universe which was a quark-gluon plasma. The understanding of this "new state of matter" would be relevant for various fields: particles physics, nuclear physics, astrophysics, cosmology...

In addition to colliding heavy ions, RHIC is able to collide protons. While these collisions do not produce quark-gluon plasma, they are interesting to physicists for other reasons. Scientists want 
to know more about the spin of particles because its origin is still unknown. Physicists have long thought that the spin of a proton was simply the sum of the spins of its three component quarks. But experiments have shown that the quarks account for only about $20 \%$ of the proton's spin. A way of unravelling the mystery is to collide "spin polarized" protons and to analyze the reaction products from the collision. RHIC is the first machine in the world with the capability to collide such beams head-on.

\section{A.3 Beam polarization and Collider-Accelerator Department}

Since 2002 polarized proton collisions occur at RHIC [13. Every year the beam polarization has been increased as it is shown by the following graph:

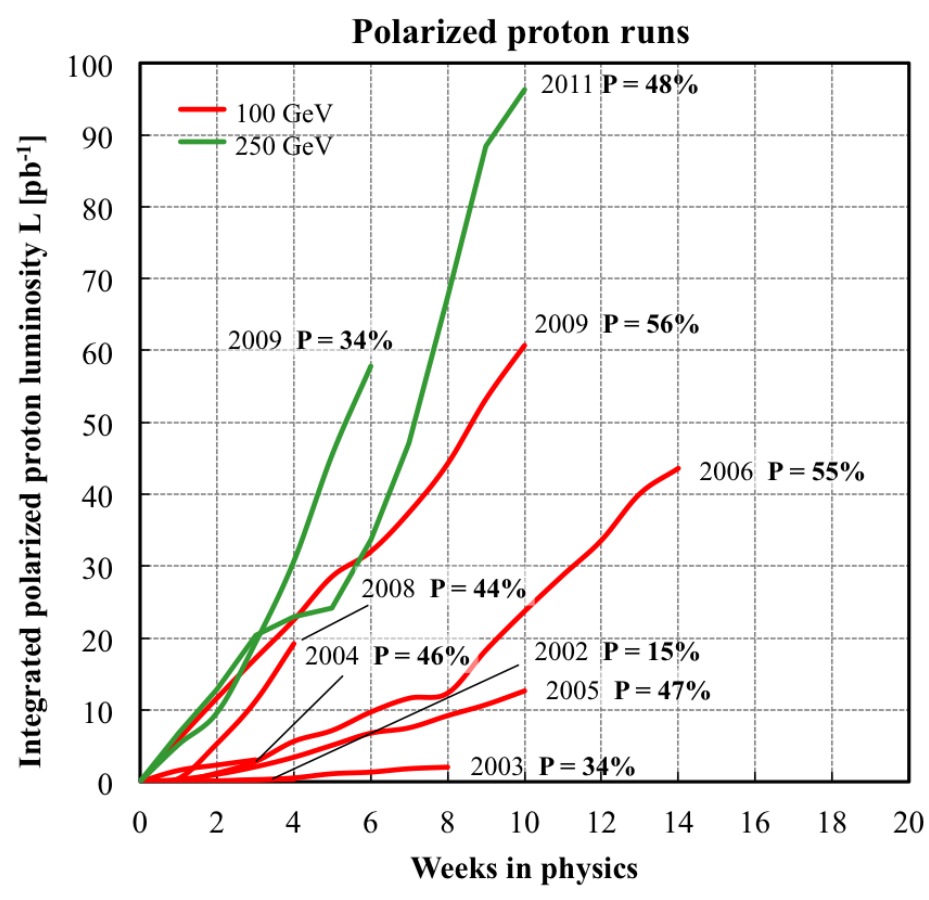

Figure 5.18: Polarized protons runs at RHIC since 2002

The beam is kept polarized during acceleration within AGS by unusual magnets called siberian snakes which are briefly presented in the next appendix. The beam polarization at interaction points has progresively been increased until reaching about $50 \%$. However the beam polarization is still too low compared to the value required for the experiments and researchers do not know why the beam polarization is lost. They know that a part of polarization is lost during the beam acceleration within the AGS. As RHIC complex is the first polarized proton collider, there is no feedback for helping researchers to improve the beam polarization. Consequently, it is still necesary to make important efforts for reaching higher values of polarization. Currently, the Collider-Accelerator Department of the BNL intents to figure it out and to correct it. Beam and spin dynamics studies aimed at reducing polarization losses within the AGS are an active part of the accelerator physics activities at C-AD. 


\section{APPENDIX B: SPIN DYNAMICS AND SIBERIAN SNAKE MAGNETS}

\section{B.1 Spin dynamics and spin depolarizing resonances}

In the presence of only magnetic fields the spin is governed by the Thomas-BMT (BARgmann, Michel, AND TELEgDI) equation [15]:

$$
\frac{d \vec{S}}{d t}=\frac{e}{\gamma m_{0}}\left(G \gamma \overrightarrow{B_{\perp}}+(1+G) \overrightarrow{B_{\|}}\right)
$$

where $\vec{S}$ is the spin vector of a particle in the frame that moves with the particle's velocity, $\overrightarrow{B_{\perp}}$ (respectively $\overrightarrow{B_{\|}}$) is the magnetic field perpendicular (respectively parralel) to the velocity vector in the laboratory rest frame. $G=\frac{g-2}{2}$ is the gyromagnetic anomaly of the proton and $\gamma$ is the Lorentz factor.

As a first approach one will consider the AGS as a perfect planar synchrotron with a vertically oriented guiding magnetic field. As a consequence of the formula 5.4, the spin vector of a proton beam precesses around the vertical axis $G \gamma$ times per orbital revolution. The number of precessions per revolution is called the spin tune:

$$
Q_{s p}=G \gamma
$$

In a real circular accelerator [16, the horizontal magnetic field $\overrightarrow{B_{x}}$ has to be taken into account. It comes from several sources, such as the vertical closed orbit and the vertical betatron oscillation, and kicks the spin vector away from the precessing around the vertical axis. Normally this perturbation is small. However, when the spin precession frequency coincides with the frequency at which the spin vector gets kicked by the horizontal magnetic field, the spin vector is kicked away coherently and a spin depolarizing resonance is encountered. A spin resonance is usually located at :

$$
Q_{s p}=k \pm l Q_{y} \pm m Q_{x} \pm n Q_{s y n}
$$

where $k, l, m$ and $n$ are integers, $Q_{x}$ and $Q_{y}$ are horizontal and vertical betatron tunes, and $Q_{s y n}$ is the tune of the synchrotron oscillation.

There are three main types of depolarizing resonances:

- The resonances at $Q_{s p}=k$, called imperfection resonances, are induced by the vertical closed orbit error. Its strength is proportional to the size of the closed orbit distortion

- The resonances at $Q_{s p}=l \pm Q_{y}$, called intrinsic resonances, come from the vertical betatron motion and are determined by the size of the vertical betatron oscillation. The larger the betatron oscillation is, the stronger the resonance is.

- The resonances at $Q_{s p}=n \pm Q_{x}$, called coupling resonances, are caused by the vertical motion with horizontal betatron frequency due to linear coupling. Its strength is proportional to the coupling coefficient in addition to the beam emittance.

The following graph represents an example of spin depolarizing resonances, the RHIC's spin depolarizing resonances spectrum: 


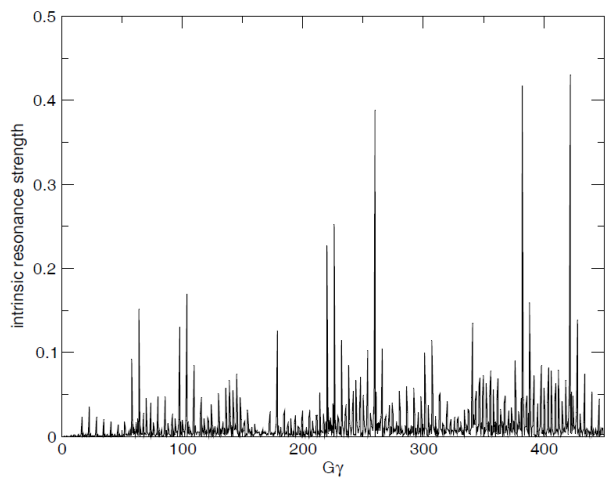

Figure 5.19: An example of spin depolarizing resonances

The beam crosses through spin depolarizing resonances during acceleration so the beam polarization could be diminished. A technique to minimize polarization loss was proposed by Derbenev and Kondratenko [18] and is to insert into the lattice special magnets called Siberian snakes magnets.

\section{B.2 Siberian snake magnets}

A siberian snake is a magnet used as a local spin rotator. The magnets' corkscrew-like design causes the direction of the magnetic field to spiral along the direction of the beam. A $100 \%$ snake achieve a $\pi$-rotation of the spin vector about an axis in the horizontal plane. Consequently, the siberian snakes are able to force the spin tune to be $1 / 2$ and thus energy independent. In this way all the imperfection, intrinsic or coupled spin resonances, can be avoided. The unusual design required for performing spin rotations is composed of wraped coils and a classical yoke. A model of the coils with the magnitude of the field superimposed on the conductor surface is given by the following graph:

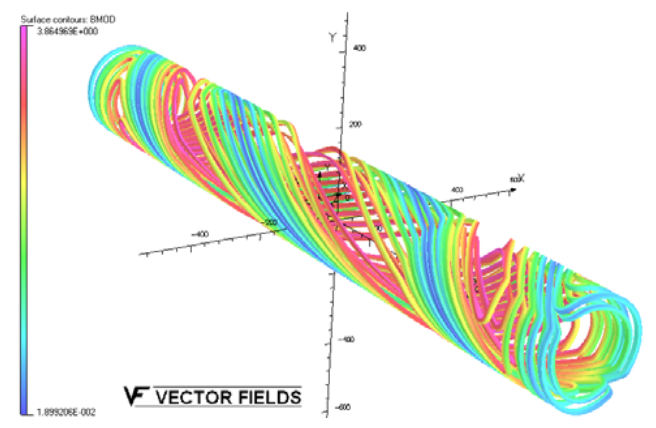

Figure 5.20: Model of the snake coils with the magnitude of the field superimposed on the conductor surface.

There are also partial snakes, 5.9\% and 10-25\% snakes, which achieve partial spin rotation. For example, in the presence of a $\Theta=0.059 \pi$ perturbation the spin tune $Q_{s p}$ will deviate from $G \gamma(5.5)$ according to [17:

$$
\cos \left(\pi Q_{s p}\right)=\cos (\pi G \gamma) \cos \left(\frac{\Theta}{2}\right)
$$

With $\Theta=0.059 \pi \simeq 0,1854$ the spin tune will never equal an integer, and thus the imperfection resonance condition is avoided completely. 
Several siberian snakes have been put in the RHIC complex in order to minimize polarization loss. Their location is presented by the following graph:

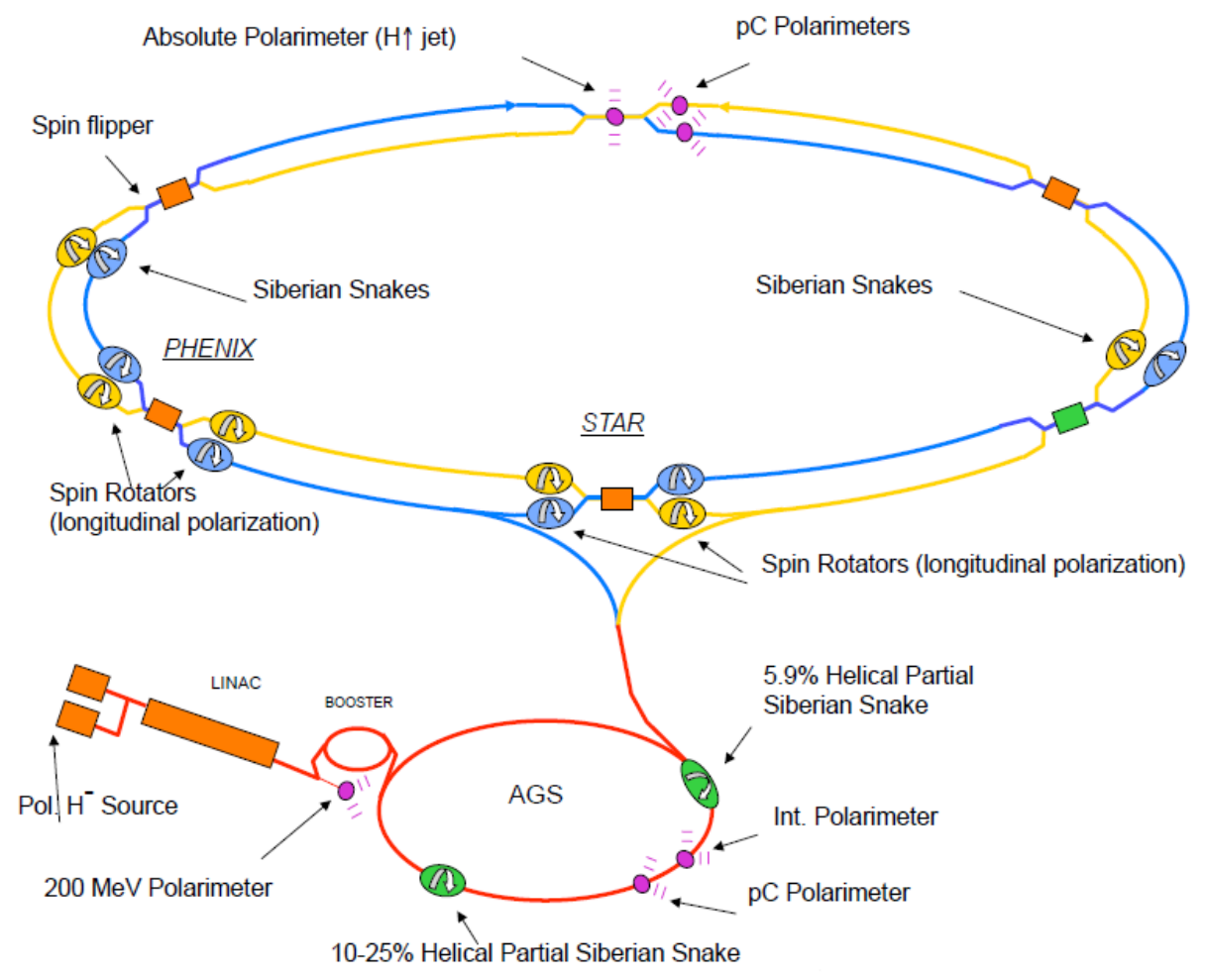

Figure 5.21: Relativistic Heavy Ion Collider complex for polarized proton beam

The helicoidal siberian snakes having been set up in the AGS in 2004 (a "6\% warm magnet snake") and 2005 (a "15\% superconducting snake") their control can still be optimized. Consequently, it is still necessary to pursue research about the beam physics within the AGS in order to improve the AGS polarization transfer efficiency. Dr. FRANÇOIS MEOT works on it since 2009 by modeling the AGS owing to the ray-tracing code $Z G O U B I$. 


\section{APPENDIX C: LIST OF VARIABLES}

- $x$ : horizontal position of a particle relative to the central orbit.

- $y$ : vertical position of a particle relative to the central orbit.

- $\vec{R}$ : position vector relative to the central orbit in the laboratory frame.

- $\vec{u}$ : particle velocity in the laboratory frame.

- $s$ : arclength along the accelerator.

- $\vec{B}$ : magnetic field.

- $m$ : mass of a particle.

- $q$ : charge of a particle.

- $\vec{v}_{0}$ : longitudinal speed of the central orbit.

- $\vec{v}$ : longitudinal speed of the particule.

- $\rho_{0}$ : radius of curvature of the central orbit.

- $\rho$ : radius of curvature of the particle trajectory.

- $K(s)$ :focusing constant of the HiLL's equation.

- $k_{d}$ : defocusing quadrupole strength.

- $k_{f}$ : focusing quadrupole strength.

- $k_{s}$ : skew quadrupole strength.

- $l_{\text {quad }}$ : quadrupole length.

- $l_{\text {skew: }}$ : skew quadrupole length.

- $\varphi$ : particle betatron phase .

- $\varphi_{0}$ : constant betatron phase determined by the initial conditions.

- $\alpha_{x}, \beta_{x}, \gamma_{x}$ : Twiss parameters along the horizontal axis in the laboratory frame.

- $Q_{x}$ : horizontal betatron tune.

- $\alpha_{y}, \beta_{y}, \gamma_{y}$ : Twiss parameters along the vertical axis in the laboratory frame.

- $Q_{y}$ : vertical betatron tune.

- $\vec{X}=\left[\begin{array}{c}x \\ x^{\prime} \\ y \\ y^{\prime}\end{array}\right]:$ phase space coordinates in the laboratory frame. $x^{\prime}$ and $y^{\prime}$ are the arclength deratives of $x$ and $y$, respectively.

- $\mathcal{T}_{0 \rightarrow 1}$ : transfer matrix from a point located at $s_{0}$ to another one located at $s_{1}$.

- $\mathcal{T}: 4 \times 4$ transfer matrix, at a point in the laboratory frame, for one turn around the system. It is also called the one-turn mapping. 
- $\mathcal{M}_{\text {uncoupled }}: 4 \times 4$ transfer matrix, at a point in the decoupled frame, for one turn around the system.

- $\mathcal{A}_{i \in\{1,2\}}: 2 \times 2$ one-dimensional transfer matrix, at a point in the decoupled frame, for one turn around the system.

- $Q_{i \in\{1,2\}}$ : eigentune i.e. tune in the decoupled frame.

- $\alpha_{i \in\{1,2\}}, \beta_{i \in\{1,2\}}$ and $\gamma_{i \in\{1,2\}}$ : generalized TwISs parameters, TwIss parameters in the decoupled frame.

- $\mathcal{V}$ : transformation matrix from the coupled frame to the decoupled one such as $\vec{X}_{\text {uncoupled }}=$ $\mathcal{V} \vec{X}_{\text {coupled }}$.

- $\mathcal{E}=\left[\overrightarrow{e_{1}}, \overrightarrow{e_{1}^{*}}, \overrightarrow{e_{2}}, \overrightarrow{e_{2}^{*}}\right]:$ normalized eigenvector of $\mathcal{T}$.

- $\lambda_{1}, \lambda_{1}^{*}, \lambda_{2}, \lambda_{2}^{*}$ : eigenvalues of $\mathcal{T}$.

- $\mathcal{D}: 4 \times 4$ diagonal transfer matrix ,at a point in the eigen frame, for one turn around the system.

- I: identity matrix.

- $r$ : coupling parameter.

- $\mathcal{C}: 2 \times 2$ coupling matrix.

- $\mathcal{C}^{+}$: complex conjugate of $\mathcal{C}$.

- $\phi_{i \in\{1,2\}}$ : one-turn phase advance of the $i$-eigenmode.

- $J_{i \in\{1,2\}}$ : constant action of the $i$-eigenmode.

- $\mathcal{R}\left(2 \pi Q_{1}, 2 \pi Q_{2}\right): 4 \times 4$ transfer matrix, at a point in the action-angle frame, for one turn around the system.

- $\mathcal{R}\left(2 \pi Q_{i \in\{1,2\}}\right): 2 \times 2$ sub-matrix of $\mathcal{R}$ along the $i$-eigenmode axis.

- $\mathcal{P}$ : transformation matrix from the action-angle frame to the laboratory frame at the entry of the lattice.

- $\mathcal{U}$ : transformation matrix from the eigenmode frame to the action-angle frame at the entry of the lattice.

- $\mathcal{U}_{i \in\{1,2\}}: 2 \times 2$ sub-matrix of $\mathcal{U}$ along the $i$-eigenmode axis.

- $\mathcal{G}$ : transformation matrix from eigenvectors matrix $\mathcal{E}$ to the matrix $\mathcal{P}$.

- $\mathcal{H}$ : multiplication matrix of $\mathcal{E}$ which is used for assuring $p_{12}=p_{34}=0$.

- $\theta_{i \in\{1,2\}}$ : phase of the eigenvector $\overrightarrow{e_{i}}$.

- $\mathcal{F}=\left[\overrightarrow{f_{1}}, \overrightarrow{f_{1}^{*}}, \overrightarrow{f_{2}}, \overrightarrow{f_{2}^{*}}\right]:$ normalized eigenvectors whom their real and imaginary part are the reference vectors of the angle-action frame.

- $\mathscr{H}_{1}$ : Hamiltonian perturbed by a weak and linear coupling.

- $L$ : length of the lattice.

- $N$ : superperiodicity of the system. 
- $C_{k, l}: k$-th coefficient of the Fourier serie for either a sum resonance $(l=+1)$ or a difference resonance $(l=-1)$.

- $a_{i \in\{x, y\}}$ : coupled motion's amplitude.

- $\psi_{i \in\{x, y\}}$ : coupled motion's phase.

- $\Delta_{k, l}$ : fractional uncoupled tune split corresponding to the $k$-th term of the Fourier serie and either a sum resonance $(l=+1)$ or a difference resonance $(l=-1)$.

- $C_{-}$: width of the tune stopband due to a linear difference coupling resonance.

- $\chi$ : phase of $C_{-}$.

- $\Delta_{-}$: fractional difference of the unperturbed tunes, i.e. the distance before reaching a linear difference coupling resonance.

- $\Delta_{+}$: fractional sum of the unperturbed tunes, i.e. the distance before reaching a linear sum coupling resonance.

- $\Delta Q_{\min }:$ minimum eigentune split.

- $G$ : gyromagnetic anomaly of the proton.

- $\gamma$ : LORENTZ factor.

- $Q_{s p}:$ spin tune.

- $Q_{\text {syn }}$ : synchrotron tune.

- $\Theta$ : spin angle perturbation induced by a siberian snake magnet. 


\section{References}

[1] E. D. Courant and H. S. Snyder, Annals of Physics 281, 360408 (2000): Theory of the AlternatingGradient Synchrotron, 15 July 1957. $1.3,4.1$

[2] D. Edwards and L. Teng, IEEE Trans. Nucl. Sci. 20, 3 (1973). 3.2, 3.3, 3.4

[3] Yun Luo, Linear coupling parametrization in the action-angle frame, 7 December 2004. 3.2. 3.4 . 3.4 .13 .4 .3

[4] Yun Luo, Matrix perturbation approach to the weak linear coupling, 28 June 2006. 3.2 $3.4,4.3$

[5] C.J Gardner, Identification of Matrix Conditions that Give Rise to the Linear Coupling Resonances, internal paper of Brookhaven National Laboratory, March 2009. 3.2 3.4

[6] Y. Luo, P. Cameron, A. Dellapenna, L. Hoff, A. Marusic, S. Peggs, and C. Schultheiss. Continuous measurement of global difference coupling using a phase-locked-loop tune meter in the Relativistic Heavy Ion Collider, 14 December 2006. 3.4 .4 .3

[7] H.Wiedemann, Section 1.3 Hamiltonian and Coupling of the Particle Accelerator Physics II, Nonlinear and Higher-Order Beam Dynamics, Springer-Verlag, Berlin, 1995. 4.1, $4.1,4.2$

[8] H.Wiedemann, Section 1.4 Hamiltonian and Coupling of the Particle Accelerator Physics II, Nonlinear and Higher-Order Beam Dynamics, Springer-Verlag, Berlin, 1995. 3.1

[9] R. Calaga and R. Tomás, Betatron coupling: Merging Hamiltonian and matrix approaches, 9 March 2005.

[10] http://en.wikipedia.org/wiki/Brookhaven_National_Laboratory, 5.6.3

[11] http://www.nsls.bnl.gov/facility/accelerator/, 5.6.3

[12] http://www.bnl.gov/rhic/complex.asp, 5.6.3

[13] http://www.agsrhichome.bnl.gov/RHIC/Runs/, 5.6.3

[14] ZGOUBI USERS' GUIDE - VERSION 4.3 - F.Méot and S.Valero CEA Saclay, DSM/DAPNIA/SEA, April 16, 2011, 2.1

[15] L.H. Thomas, Philos. Mag. 3, 1 (1927); V. Bargmann, L. Michel, and V.L. Telegdi, Phys. Rev. Lett. 2. 435 (1959), 5.6.3

[16] http://www.agsrhichome.bnl.gov/RHIC/Runs/RhicRun11pp_1.pdf, 5.6.3

[17] Vahid H. Ranjbar, Increasing proton polarization in AGS and RHIC, October 16, 2002, Indiana University. 5.6.3

[18] Ya.S. Derbenev and A.M. Kondratenko, Zh. Eksp. Teor. Fiz. 62, 430 (1972) [Sov. Phys. JETP 35, 230 (1972)]; Ya.S. Derbenev et. al., Part. Accel. 8, 115 (1978). 5.6.3 


\section{SUMMARY}

During this 5-months internship the weak and linear coupling occuring in particle accelerators was studied. It was noticed owing to ray-tracing simulations made by ZGOUBI that the uncoupled formalism needs to be generalized for treating with $Z G O U B I$ the one-turn mapping of coupled systems. As a consequence a mathematical formalism treating the coupling was proposed in order to implement it into ZGOUBI: the EDWARDS and TENG's parametrization. It introduced the EDWARDS and TENG's parameters which corresponds, on one hand, to the generalized Twiss parameters and, on another hand, to the coupling parameters $r$ and $\mathcal{C}$.

One developed both physical and mathematical analyses for helping the interpretation of these new parameters and the understanding of future simulations. It required to use both a matrix approach and a perturbation Hamiltonian theory defining new variables such as the coupling strenght $\left|C_{-}\right|$as well as the tune distance from a linear coupling resonance $\Delta_{-/+}$and the unperturbed tunes $Q_{x}^{0}$ and $Q_{y}^{0}$. It has been derived relations between these two approaches for the weak linear difference resonances.

New simulations have been handled with the ET's parametrization implemented into ZGOUBI for some benchmark systems. First, it came out a better precision of eigentune computations, it was reduced from $10^{-2}$ to $10^{-6}$. Then it was demonstrated that the unperturbed tunes and so the tune distance from the linear coupling resonances are computed with a good accuracy. Nevertheless one found out that the coupling strength established by the Hamiltonian perturbation approach is only valid at the vicinity of the linear difference resonances. Finally the propagation of the generalized Twiss parameters along the ring was tested with a model of the Alternating Gradient Synchrotron and was in good agreement with the MADX computations. The coupling parameter $r$ was also transported along the ring. First it showed that $r$ is not a periodic function then it exhibited that $r$-jumps hint the position of the coupling sources in the AGS: the siberian snake magnets.

\section{RÉSUMÉ}

Le sujet de ce stage a été l'étude du couplage faible et linéaire des oscillations betatron transverses au sein des accélérateurs de particules. Au cours de simulations réalisées avec ZGOUBI, un programme de tracé de trajectoires, on a constaté que le formalisme non-couplé qu'il utilise n'est pas approprié pour traiter la matrice du tour des systèmes couplés et doit être généralisé. Par conséquent, un formalisme capable d'analyser le mouvement couplé a été proposé dans l'objectif de l'implémenter au sein de ZGOUBI : la paramétrisation d'EDwards et TENG. Ce formalisme définit des fonctions de Twiss généralisées et les paramètres de couplage $r$ et $\mathcal{C}$.

Des analyses physiques et mathématiques de ces nouveaux paramètres ont ensuite été développées afin de donner des outils visant à faciliter l'interprétation de résultats de futures simulations. Pour cela, il a fallu développer en parralèle une approche matricielle et une théorie de l'Hamiltonien perturbé. Cette dernière définit de nouvelles variables : la force du couplage $\left|C_{-}\right|$, les distances en nombre d'onde $\Delta_{-/+}$séparant le système d'une résonance linéaire de couplage, les nombres d'onde non-perturbés $Q_{x}^{0}$ et $Q_{y}^{0}$. Puis on a explicité des relations mathématiques entre ces deux approches pour des systèmes proches de résonances de couplage dîtes de différences.

On a ensuite réalisé de nouvelles simulations de systèmes de référence avec la paramétrisation ET implémenté dans $Z G O U B I$. On a montré, premièrement, que la précision sur le calcul des nombres d'onde propres est passée de $10^{-2}$ à $10^{-6}$, secondement, que les $Q_{x, y}^{0}$ et les $\Delta_{-/+}$sont calculés avec une bonne précision. Cependant, on a trouvé que le calcul de la force de couplage défini par la théorie de l'Hamiltonien perturbé n'est valide qu'au voisinage de resonances de différence. Finalement, on a simulé la propagation des paramètres de ET le long d'une machine: l'Alternating Gradient Synchrotron. Les fonctions de Twiss généralisées sont en accord avec celles calculées par MADX. Le paramètre de couplage $r$ a aussi été transporté le long de l'anneau. Il a été constaté d'une part que $r$ n'est pas une fonction périodique et d'autre part que les sauts de $r$ se situent aux sources de couplage de l'AGS: les serpents sibériens. 